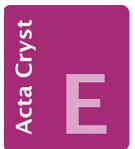

CRYSTALLOGRAPHIC COMMUNICATIONS

ISSN 2056-9890
Received 15 April 2020

Accepted 6 May 2020

Edited by B. Therrien, University of Neuchâtel, Switzerland

Keywords: crystal structure; co-crystal; Hirshfeld surface analysis; 3,4-ethylenedioxythiophene; EDOT.

CCDC reference: 2001277

Supporting information: this article has supporting information at journals.iucr.org/e

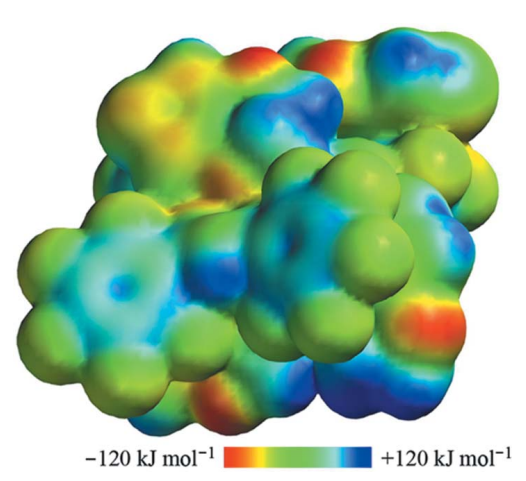

OPEN $\odot$ ACCESS

\section{Co-crystal structure, Hirshfeld surface analysis and DFT studies of 3,4-ethylenedioxythiophene solvated bis[1,3-bis(pentafluorophenyl)propane-1,3- dionato]copper(II)}

\author{
Yusuke Habuka, ${ }^{\text {a Emily Ami Takeuchi }}{ }^{\mathrm{a}, \mathrm{b}}$ and Akiko Hori ${ }^{\mathrm{a} *}$
}

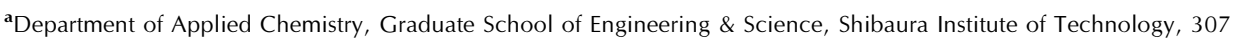
Fukasaku, Minuma-ku, Saitama-shi, Saitama 337-8570, Japan, and ${ }^{\mathbf{b} C e n t e r}$ for Natural and Human Sciences (CCNH), Federal University of ABC, Santo Andre, Sao Paulo 09210-580, Brazil. *Correspondence e-mail: ahori@shibaura-it.ac.jp

The title complex, $\mathrm{Cu}(L)_{2}$ or $\left[\mathrm{Cu}\left(\mathrm{C}_{15} \mathrm{HF}_{10} \mathrm{O}_{2}\right)_{2}\right]$, comprised of one copper ion and two fully fluorinated ligands $\left(L^{-}\right)$, was crystallized with 3,4-ethylenedioxythiophene (EDOT, $\mathrm{C}_{6} \mathrm{H}_{6} \mathrm{O}_{2} \mathrm{~S}$ ) as a guest molecule to give in a dichloromethane solution a unique co-crystal, $\mathrm{Cu}(L)_{2} \cdot 3 \mathrm{C}_{6} \mathrm{H}_{6} \mathrm{O}_{2} \mathrm{~S}$. In the crystal, the oxygen of one guest molecule, EDOT-1, is coordinated to the metal to give an alternate linear arrangement, and the $\pi$-planes of the others, EDOT-2 and EDOT-3, interact weakly with the pentafluorophenyl groups of the complex through areneperfluoroarene interactions. Head-to-tail columnar and head-to-head dimeric arrangements are observed for EDOT-2 and EDOT-3, respectively, in the crystal. The Hirshfeld surface analysis indicated that the most important contributions for the crystal packing are from the F $\cdots \mathrm{F}(20.4 \%), \mathrm{F} \cdots \mathrm{H} / \mathrm{H} \cdots \mathrm{F}$ $(24.5 \%)$ and $\mathrm{F} \cdots \mathrm{C} / \mathrm{C} \cdots \mathrm{F}(9.6 \%)$ interactions. The density functional theory (DFT) optimized structure at the $\omega$ B97X-D 6-31G* level was compared with the experimentally determined molecular structure in the solid state.

\section{Chemical context}

3,4-Ethylenedioxythiophene, EDOT, is a familiar reagent for polythiophene or oligothiophene organic-active materials such as organic conductive macromolecules and optoelectronic materials. The corresponding poly-3,4-ethylenedioxythiophene, PEDOT, is one of the typical organic conductive materials with a high conductivity, environmental stability, mechanical strength and visible light transmittance, thus showing wide ranges of applications (Skotheim et al., 1998; Groenendaal et al., 2000; Kirchmeyer \& Reuter, 2005). The affinity as a guest molecule and the corresponding intermolecular interactions in co-crystals of EDOT are crucial issues for chemists in order to understand the molecular recognition and supramolecular association events (Storsberg et al., 2000). The crystal packing and the relative intermolecular interactions are estimated by the oxygen and sulfur atoms for coordination bonds and molecular stacking of the $\pi$ interactions for the five-membered hetero-conjugated aromatic ring. On the other hand, molecular crystals of fully fluorinated coordination complexes have been studied as hosts, showing flexible and responsive crystal-packing structures depending on the guest molecules. Typically, the copper complex, $\mathrm{Cu}(L)_{2}$, produces unique co-crystals abundantly taken into benzene derivatives after crystallization and 
reversibly encapsulates their vapors (Hori et al., 2014), while the corresponding single crystals of $\mathrm{Cu}(\mathrm{dbm})_{2}(\mathrm{dbm}=\mathrm{di}-$ benzoylmethane) showed no interaction with the guest molecules. The driving forces of the molecular recognition estimated a metal $\cdots \pi$ interaction (Hunter, 1994; Ma \& Dougherty, 1997) induced by improvement of the cationic properties of the central metal as a result of the fluorinewithdrawing nature and arene-perfluoroarene interaction (Williams, 1993, 2017; Hori, 2012) induced by the exact opposite quadrupole moment between the pentafluorophenyl ring of the complex and the aromatic ring of the guest molecule.

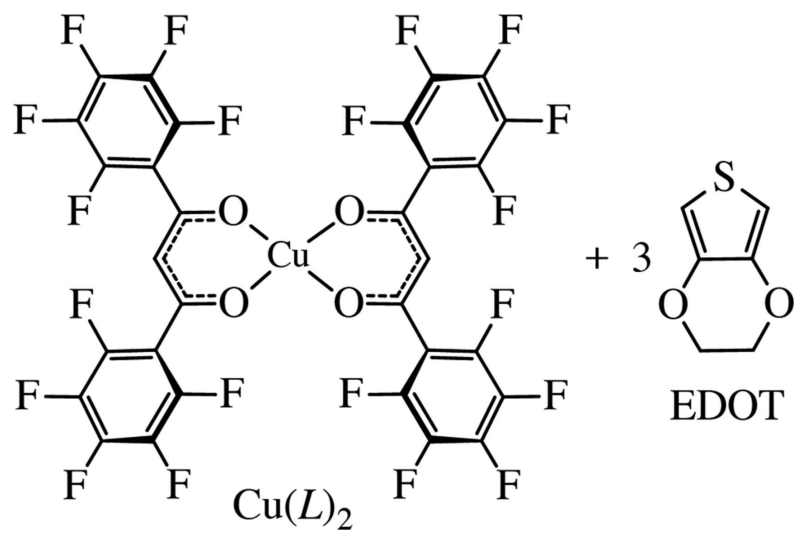

In this study, we examined the encapsulation of 3,4-ethylenedioxythiophene for the title complex, $\mathrm{Cu}(L)_{2}$, indicating a new guest-encapsulated crystal, $\mathrm{Cu}(L)_{2} \cdot 3 \mathrm{EDOT}(\mathrm{I})$, as shown in the Scheme. The crystal of (I) was prepared by previously reported protocols (Hori \& Arii, 2007). Typically, $\mathrm{Cu}(L)_{2}$ and an excess amount of EDOT in $\mathrm{CH}_{2} \mathrm{Cl}_{2}$ (or $\mathrm{AcOEt}$ ) were slowly evaporated to yield green block-shaped crystals. The driving forces and the detailed weak intermolecular inter-

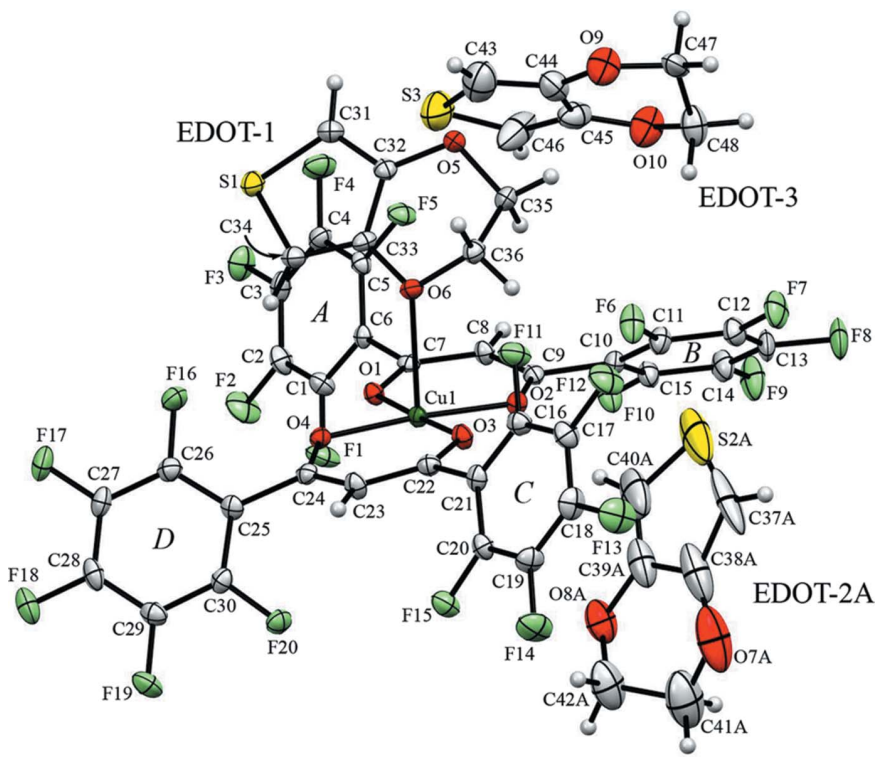

Figure 1

The molecular structure of (I) at $100 \mathrm{~K}$, showing the atom-labeling scheme. Displacement ellipsoids are drawn at the $50 \%$ probability level. The minor EDOT- $2 B$ component is omitted. actions were investigated by Hirshfeld surface analysis and DFT calculations. Using the same procedure, the corresponding compound $\operatorname{Pd}(L)_{2} \cdot n$ EDOT was not obtained, then $\operatorname{Pd}(L)_{2}$ was separately crystallized, showing different metal characteristics and affinity for EDOT. The electrostatic potential of the metal ions is also discussed.

\section{Structural commentary}

The asymmetric unit of (I) contains one entire complex molecule and three EDOT molecules. The complex is noncentrosymmetric and comprises one $\mathrm{Cu}^{2+}$ ion and two ligands $(L)$ to give a mononuclear $\mathrm{Cu}^{2+}$ complex, as shown in Fig. 1. The geometry around the metal center is pseudo-square planar; the bond distances $\mathrm{Cu} 1-\mathrm{O} 1, \mathrm{Cu}-\mathrm{O} 2, \mathrm{Cu}-\mathrm{O} 3$ and Cu1-O4 are 1.940 (2), 1.941 (2), 1.922 (2) and 1.928 (2) A, respectively. The pentafluorophenyl groups [rings $A-D(\mathrm{C} 1-$ $\mathrm{C} 6, \mathrm{C} 10-\mathrm{C} 15, \mathrm{C} 16-\mathrm{C} 21$ and $\mathrm{C} 25-\mathrm{C} 30$, respectively)] are highly twisted with respect to the coordination plane; the dihedral angle between ring $A$ (or ring $B$ ) and $\mathrm{Cu} 1 / \mathrm{O} 1 / \mathrm{C} 7-\mathrm{C} 9 / \mathrm{O} 2$ is $65.80(13)^{\circ}$ [or $36.24(15)^{\circ}$ ] and the dihedral angle between ring $C$ (or ring $D$ ) and $\mathrm{Cu} 1 / \mathrm{O} 3 / \mathrm{C} 22-\mathrm{C} 24 / \mathrm{O} 4$ is $54.97(14)^{\circ}$ [or $\left.51.22(13)^{\circ}\right]$, indicating that all these rings are crystallographically different. The flexible and twisted rings allow intermolecular interactions with the EDOT molecules to consolidate the crystal of (I). The oxygen atoms of EDOT-1 are coordinated with atom $\mathrm{Cu} 1$ of the complex molecule; the lengths of the coordination bonds are 2.421(2) and 2.711 (2) $\AA$ for $\mathrm{Cu} 1-\mathrm{O} 6$ and $\mathrm{Cu} 1-\mathrm{O}^{\mathrm{i}}$ [symmetry code: (i) $x+1, y, z]$, respectively (Figs. 1 and $2 a$ ). The EDOT-2 molecule shows disorder, the occupancy of the major component, EDOT-2 $A$, being 0.691 (4); EDOT-2 $A$ shows close interactions with ring $C$ of $\mathrm{Cu}(L)_{2}$ through an arene-perfluoroarene interaction. The EDOT-3 molecule shows no remarkable interactions in the crystal packing as discussed below. Each EDOT molecule shows a $\pi$-localized structure as shown in the Scheme; the lengths of the $\mathrm{C}=\mathrm{C}$ double bonds are 1.355 (5) and 1.351 (4) $\AA$ for EDOT-1, 1.46 (1) and

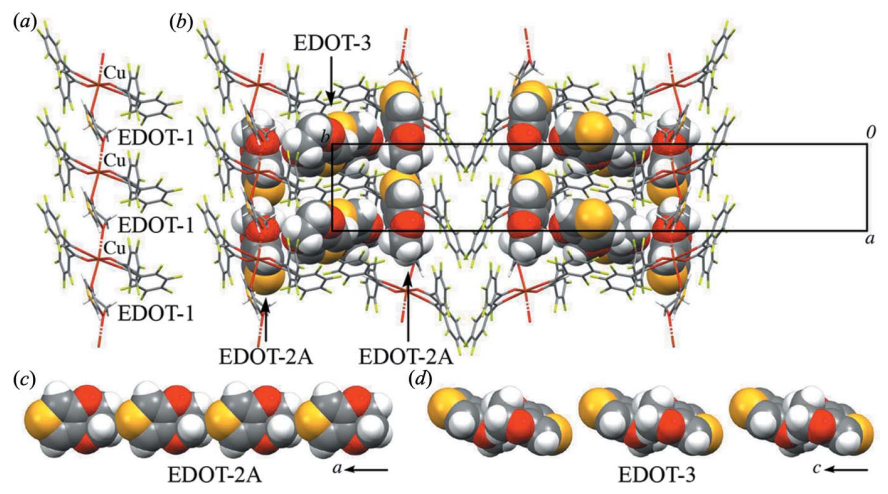

Figure 2

Views of part of the crystal structure of (I): (a) 1:1 alternating linear structure with EDOT-1 and $\mathrm{Cu}(L)_{2},(b)$ EDOT-2A and EDOT-3 in the void spaces of the linear chain with the $(c)$ head-to-tail and $(d)$ head-tohead arrangements in the crystal. Color scheme: $\mathrm{C}$, gray; $\mathrm{H}$, white; $\mathrm{Cu}$, orange; F, light green; $\mathrm{O}$, red; $\mathrm{S}$, yellow. 
Table 1

Hydrogen-bond geometry $\left(\AA,^{\circ}\right)$.

\begin{tabular}{lllll}
\hline$D-\mathrm{H} \cdots A$ & $D-\mathrm{H}$ & $\mathrm{H} \cdots A$ & $D \cdots A$ & $D-\mathrm{H} \cdots A$ \\
\hline $\mathrm{C} 23-\mathrm{H} 23 \cdots \mathrm{F} 17^{\mathrm{i}}$ & 0.95 & 2.41 & $3.362(4)$ & 179 \\
$\mathrm{C} 31-\mathrm{H} 31 \cdots \mathrm{O} 1^{\mathrm{ii}}$ & 0.95 & 2.57 & $3.351(4)$ & 139 \\
$\mathrm{C} 35-\mathrm{H} 35 A \cdots \mathrm{O} 8 B^{\mathrm{ii}}$ & 0.99 & 2.45 & $3.349(16)$ & 151 \\
$\mathrm{C} 37 A-\mathrm{H} 37 A \cdots \mathrm{S} 1^{\text {ii }}$ & 0.95 & 2.77 & $3.590(9)$ & 145 \\
$\mathrm{C} 41 A-\mathrm{H} 41 A \cdots \mathrm{S} 2 A^{\text {iv }}$ & 0.99 & 2.51 & $3.051(11)$ & 114 \\
$\mathrm{C} 42 A-\mathrm{H} 42 A \cdots \mathrm{S} 2 A^{\text {iv }}$ & 0.99 & 2.57 & $3.220(9)$ & 123 \\
$\mathrm{C} 42 A-\mathrm{H} 42 A \cdots \mathrm{F} 6^{\text {iv }}$ & 0.99 & 2.45 & $3.162(8)$ & 128 \\
$\mathrm{C} 48-\mathrm{H} 48 B \cdots \mathrm{F} 10^{v}$ & 0.99 & 2.51 & $3.326(5)$ & 140 \\
\hline
\end{tabular}

Symmetry codes: (i) $x,-y+\frac{3}{2}, z-\frac{1}{2}$; (ii) $x-1, y, z$; (iii) $x, y, z-1$; (iv) $x+1, y, z$; (v) $-x+1,-y+1,-z+1$.

1.32 (1) $\AA$ for EDOT-2A, and 1.361 (6) and 1.365 (6) $\AA$ for EDOT-3. EDOT-2A has a large variation in the distance because of the structural disorder, while the analysis was performed without restricting the binding distance of the carbon-to-carbon bonds. For comparison of the molecular recognitions of $\mathrm{Cu}(L)_{2}$, negative quadrupole moments of the molecules, e.g., benzene and carbon dioxide, are reversibly recognized in the crystals, because of the positive quadrupole moments of the pentaflurophenyl groups (Hori et al. 2014, 2017). Thus, the crystal structure of (I) indicates the possibility that the butadiene moiety, $\mathrm{C}=\mathrm{C}-\mathrm{C}=\mathrm{C}$, in EDOT also has a negative surface and interacts in the crystal of $\mathrm{Cu}(L)_{2}$ through electrostatic interactions.

\section{Supramolecular features}

The partial view of the packing structure in Fig. $2 a$ clearly shows a one-dimensional linear chain orientation between the complex molecule and EDOT-1. EDOT-1 coordinates to the copper ion of the complex to form a 1:1 alternating linear structure along the $a$-axis direction. The EDOT- $2 A$ and EDOT-3 molecules are inserted in the voids of the linear chain along the $a$ - and $c$-axis directions, respectively. EDOT- $2 A$ forms a head-to-tail one-dimensional chain (Fig. $2 c$ ) with weak hydrogen bonds (Table 1 ) between the sulfur atom and the aliphatic proton with $D \cdots A$ distances of 3.051 (11) and $3.220(9) \AA \quad$ for $\mathrm{C} 41 A-\mathrm{H} 41 A \cdots \mathrm{S} 2 A$ and $\mathrm{C} 42 A-$ $\mathrm{H} 42 A \cdots \mathrm{S} 2 A$, respectively, and the molecule is further sandwiched by the pentafluorophenyl rings of the complex. EDOT3 forms discrete dimers (Fig. $2 d$ ) in a head-to-head configuration between the aliphatic moieties, and the dimers are also surrounded by the pentafluorophenyl rings of the complex molecule. Short intermolecular interactions between the centroids $(\mathrm{Cg})$ of the pentafluorophenyl ring in $\mathrm{Cu}(L)_{2}$ and the five-membered ring of EDOT are observed. The pentafluorophenyl ring $A$ (C1-C6) is sited on the adjacent EDOT$2 A^{\text {ii }}$ (S2A/C $37 A-\mathrm{C} 40 A$ ) [symmetry code: (ii) $x, y, z+1$ ]: the centroid-centroid distance $C g \cdots C g$ is 3.950 (4) $\AA$ and the shortest perpendicular distance of $C g$ (ring $A$ ) on the ring of EDOT- $2 A^{\mathrm{ii}}$ is $3.0832(13) \AA$. Ring $B(\mathrm{C} 10-\mathrm{C} 15)$ is sandwiched between two adjacent molecules, EDOT- $3^{\text {iii }}$ and EDOT- $3^{\text {iv }}$ (S3/C43-C46) [symmetry code: (iii) $-x,-y+1,-z+1$; (iv) $-x+1,-y+1,-z+1]$ : the centroid-centroid distances are
3.906 (2) and 4.054 (2) $\mathrm{A}$, respectively, and the corresponding shortest perpendicular distances are $3.5236(19)$ and 3.2687 (15) $\AA$, respectively. Ring $C$ (C16-C21) interacts with EDOT-2 $A(\mathrm{~S} 2 A / \mathrm{C} 37 A-\mathrm{C} 40 A)$ and EDOT-2B (minor disorder component; S2B/C37B-C40B); the centroid-centroid distances are 3.586 (3) and 3.684 (5) $\AA$, respectively, and the corresponding shortest perpendicular distances are 3.5337 (14) and 3.299 (4) A, respectively. Ring D (C25-C30) interacts with the adjacent EDOT- ${ }^{\mathrm{i}}$ (S1/C31-C34) with centroid-centroid and perpendicular distances of 3.7052 (19) and 3.3405 (13) $\AA$, respectively. The results indicate that a remarkable arene-perfluoroarene interaction is observed for EDOT- $2 A$ with a length close to the sum of the van der Waals radii. A notable intramolecular $\mathrm{C}-\mathrm{F} \cdots \pi$ interaction is observed between F5 and EDOT-1 [3.287 (2) Å] and intermolecular $\mathrm{C}-\mathrm{F} \cdots \pi$ interactions occur between the pentafluorophenyl rings as an F..$\pi$ (hole) interaction; the distances

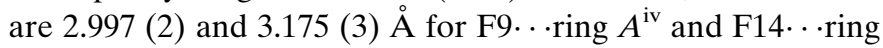
$D^{\mathrm{v}}$, respectively [symmetry code: (v) $x,-y+\frac{3}{2}, z-\frac{1}{2}$ ]. These aromatic interactions are estimated to be induced by the positive electron distribution and quadrupole moment of the pentafluorophenyl rings.

\section{Hirshfeld surface analysis}

To understand all the intermolecular interactions, a Hirshfeld surface (HS) analysis (Hirshfeld, 1977; Spackman \& Jayatilaka, 2009) was carried out using Crystal Explorer 17.5 (Turner et al., 2017). The HS of the complex molecule mapped with $d_{\mathrm{e}}$ (the distance between the surface and external atoms) and the corresponding fingerprint plots are shown in Figs. 3 and 4, respectively. The complex $\mathrm{Cu}(L)_{2}$ is surrounded by EDOT and $\mathrm{Cu}(L)_{2}$ molecules and the intermolecular interactions are indicated in red (Fig. 3). The main interactions for the whole

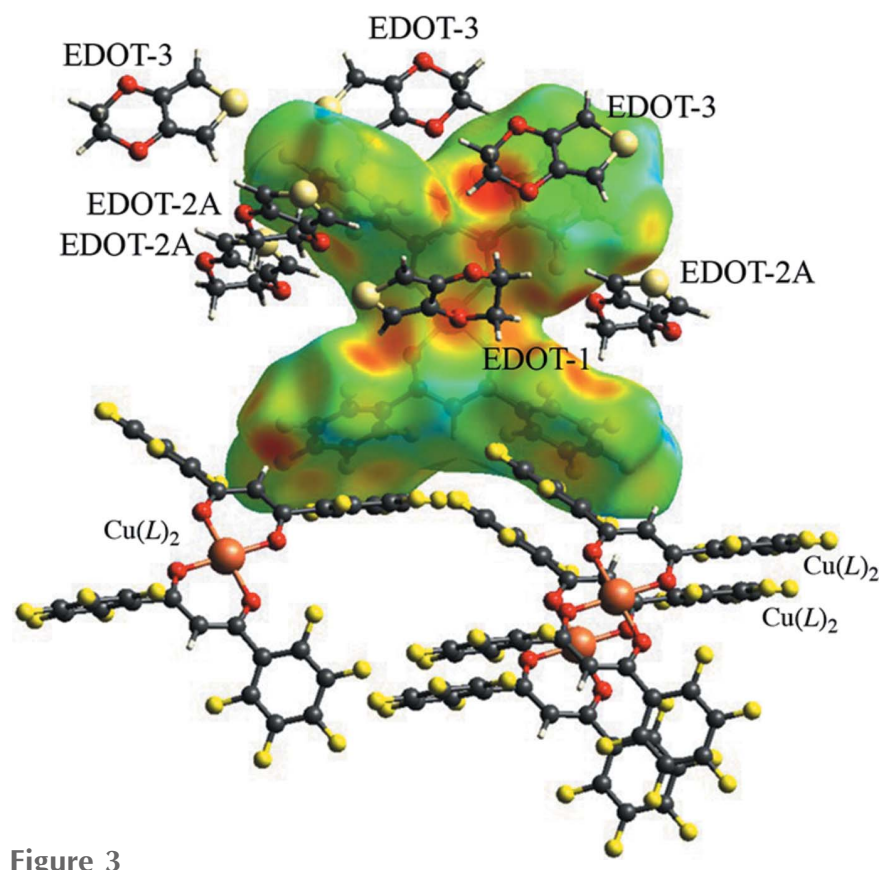

HS of the complex mapped with $d_{\mathrm{e}}$. 

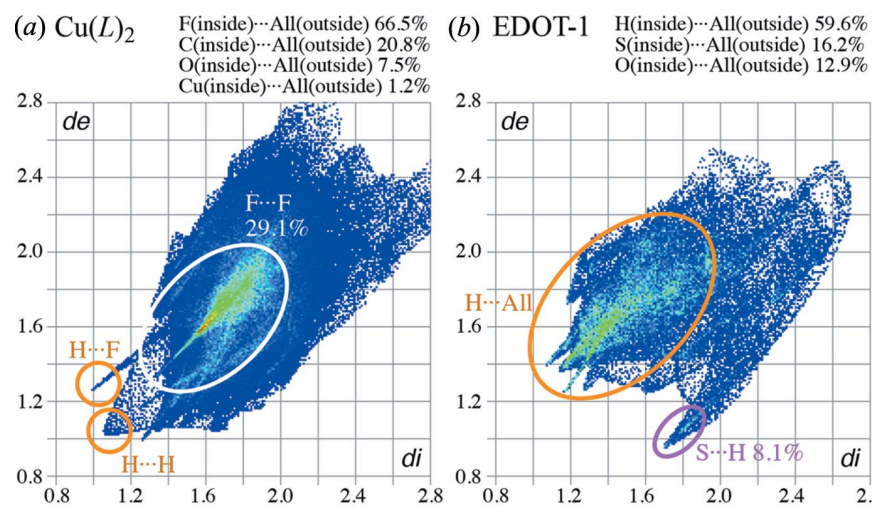

(c) EDOT-2A $\begin{array}{r}\mathrm{H} \text { (inside) } \cdots \text { All(outside) } \\ \text { O(inside) } \cdots \text { All (outside) } 16.2 \%\end{array}$
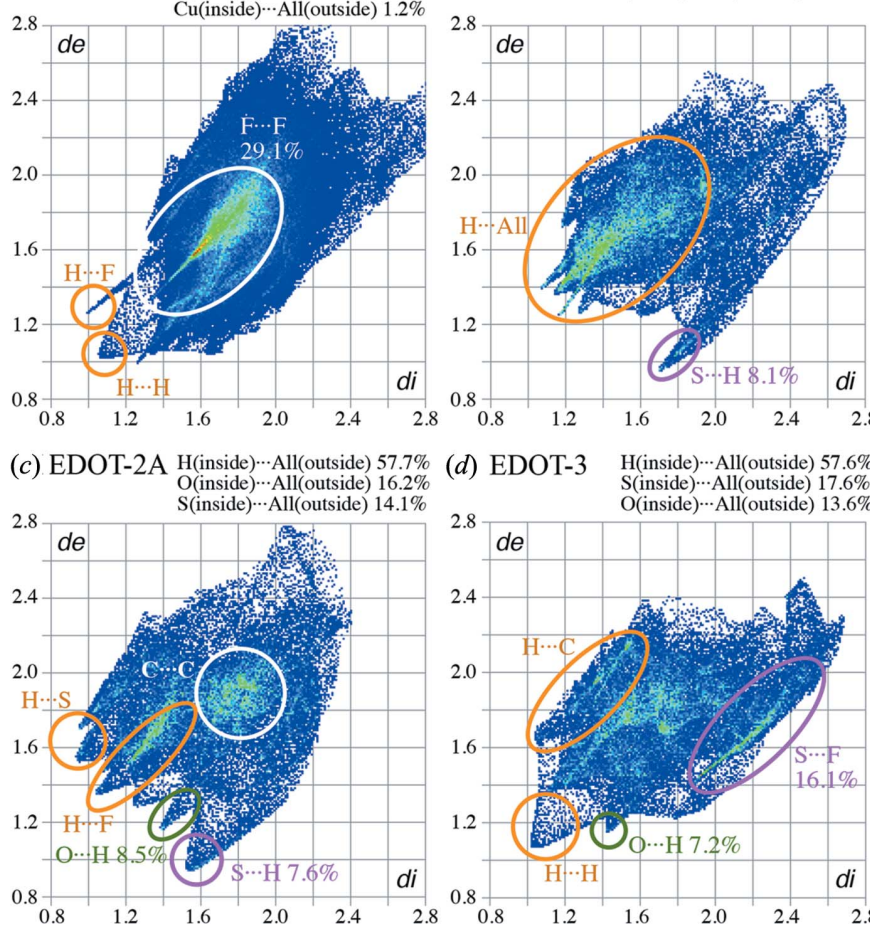

(d) EDOT-3 H(inside) $\cdots$ All(outside) $57.6 \%$
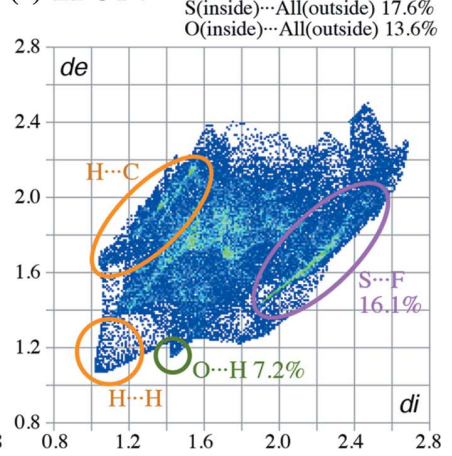

Figure 4

Fingerprint plots for the $\mathrm{Cu}(L)_{2}$ and EDOT molecules in (I).

structure are $\mathrm{F} \cdots \mathrm{F}$ and $\mathrm{F} \cdots \mathrm{H} / \mathrm{H} \cdots \mathrm{F}$, contributing $20.4 \%$ and $24.5 \%$, respectively, to the overall crystal packing due to the high surface area of fluorine for the complex. The presence of $\pi-\pi$ and $\mathrm{C}-\mathrm{H} \cdots \pi$ interactions is reflected in the contributions of the $\mathrm{C} \cdots \mathrm{C}(5.2 \%)$ and $\mathrm{C} \cdots \mathrm{H} / \mathrm{H} \cdots \mathrm{C}(6.2 \%)$ contacts. The two-dimensional fingerprint plots (McKinnon et al., 2007) of the independent $\mathrm{Cu}(L)_{2}$ and three EDOT molecules are shown in Fig. $4 a-d$, together with the contributions of each element. For $\mathrm{Cu}(L)_{2}$, the contribution of the $\mathrm{Cu}$ atom indicates interaction only with the oxygen of EDOT-1 (1.2\%). For the three EDOT molecules, the main interactions are $\mathrm{H} \cdots \mathrm{F}$ contributing $23.6 \%, 25.3 \%$, and $26.8 \%$ for EDOT-1, $2 A$ and 3 , respectively. The contribution of the $\pi-\pi$ interactions through C. . C interactions shows the relationship EDOT-2A (8.2\%) > EDOT-3 (6.0\%) > EDOT-1 (4.5\%), which indicates good agreement of the arene-perfluoroarene interactions in the crystal packing. For the sulfur in EDOT, the $\mathrm{S} \cdots \mathrm{H}$ interaction is observed for EDOT-1 $(8.1 \%)>$ EDOT-2A $(7.6 \%)$, but no interaction for EDOT-3 $(0.0 \%)$ and the S $\cdots \mathrm{F}$ interaction is observed for EDOT-3 (16.1\%) >> EDOT-2A (4.3\%) > EDOT$1(3.3 \%)$, which is also shown by the relationships of Figs. 2 and 3. For the oxygen in EDOT, $\mathrm{O} \cdots \mathrm{H}$ interactions are observed [EDOT-2A (8.5\%) > EDOT-3 (7.2\%) > EDOT-1 $(2.0 \%)]$ as well as $\mathrm{O} \cdots \mathrm{F}$ [EDOT-2A $(6.1 \%)>$ EDOT-3 $(2.2 \%)$ $>$ EDOT-1 $(1.2 \%)$ ] and O $\cdots$ Cu interactions [EDOT-1 $(4.5 \%)$ $>$ EDOT- $2 A$ and $3(0.0 \%)$ ]. These results indicate that the main intermolecular contributions without $\pi$-interactions are $\mathrm{Cu} \cdots \mathrm{O}$ and $\mathrm{S} \cdots \mathrm{H}$ for EDOT-1, O $\cdots \mathrm{H}$ for EDOT-2A, and S...F for EDOT-3.

\section{DFT calculations}

The DFT calculations were performed to obtain quantitative values for the surface potential and intermolecular interactions. The electrostatic potentials of $\mathrm{Cu}(L)_{2}$ and EDOT in (I) range from -135.79 to $+162.31 \mathrm{~kJ} \mathrm{~mol}^{-1}$, as shown in Fig. 5 . The highest electrostatic potential, in which the electron-poor region is shown in blue, is on the $\mathrm{Cu}$ atom, the edge of the ketonato hydrogen, the central part of the pentafluorophenyl rings in $\mathrm{Cu}(L)_{2}$, and the aromatic and aliphatic hydrogen atoms of EDOT. The lowest electrostatic potential, shown in red, is around the oxygen atoms of $\mathrm{Cu}(L)_{2}$ and EDOT. The highest electrostatic potentials of the centers of the pentafluorophenyl rings $A-D$ are approximately $+97,+90,+91$, $+83 \mathrm{~kJ} \mathrm{~mol}^{-1}$, respectively, which is almost the same as the independently calculated value for $\mathrm{Cu}(L)_{2}\left(+97 \mathrm{~kJ} \mathrm{~mol}^{-1}\right.$ for the pentafluorophenyl ring), which was calculated using the currently reported crystal structure (Crowder et al., 2019). The lowest electrostatic potentials of the five-membered rings of EDOT are $-77,-63$, and $-63 \mathrm{~kJ} \mathrm{~mol}^{-1}$ for EDOT-1, $2 A$ and 3 , respectively, indicating the electron distribution is slightly lower than that calculated independently for EDOT $\left(-81 \mathrm{~kJ} \mathrm{~mol}^{-1}\right)$ and used to estimate the intermolecular interactions of $\mathrm{Cu}(L)_{2}$ and EDOT. The electrostatic potential maps of the EDOT molecules are shown in Fig. 5c. The lefthand structure, optimized and calculated for an independent molecule, clearly indicates that the EDOT- $2 A$ has more positive surfaces. The lowest electrostatic potentials of the oxygen atoms are -117 and $-118 \mathrm{~kJ} \mathrm{~mol}^{-1}$ for EDOT (calculated from the refined structure of a single component), -85 and $-121 \mathrm{~kJ} \mathrm{~mol}^{-1}$ for EDOT-1, -109 and $-63 \mathrm{~kJ} \mathrm{~mol}^{-1}$ for EDOT-2A, and -102 and $-113 \mathrm{~kJ} \mathrm{~mol}^{-1}$ for EDOT-3. These values show the strength of the intermolecular inter-

(a)

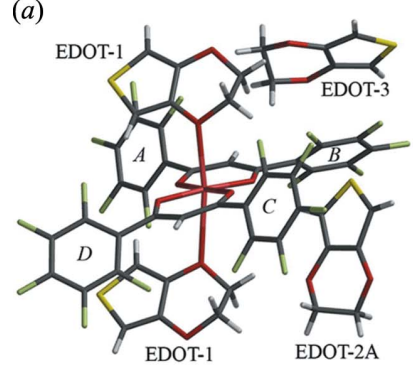

(c)
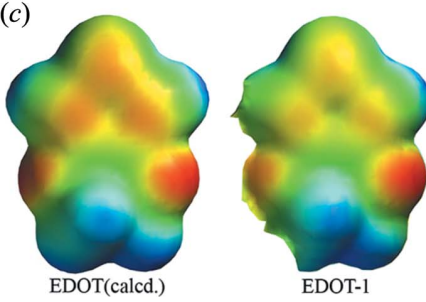

Figure 5

(a) Structure and (b) the energy potential maps of $\mathrm{Cu}(L)_{2}$ with the surrounding EDOT molecules and $(c)$ the energy potential maps of independent EDOT and each solvated EDOT molecule in (I). The color of the potential is shown between $-120 \mathrm{~kJ} \mathrm{~mol}^{-1}$ (red) to $+120 \mathrm{~kJ} \mathrm{~mol}^{-1}$ (blue). 
actions of the oxygen atoms; one oxygen in EDOT-1 is an electron donor for the coordination bond with decreasing electron density $\left(-85 \mathrm{~kJ} \mathrm{~mol}^{-1}\right)$ and one oxygen in EDOT- $2 A$ is an electron donor for the hydrogen bond with decreasing electron density $\left(-63 \mathrm{~kJ} \mathrm{~mol}^{-1}\right)$. The highest electrostatic potential of the surface of the aliphatic $\mathrm{H}$ atoms is $+162 \mathrm{~kJ} \mathrm{~mol}^{-1}$ in EDOT- $2 A$ and the values of each EDOT are $+116,+112$, and $+123 \mathrm{~kJ} \mathrm{~mol}^{-1}$ for EDOT (calculated), EDOT-1, and EDOT-3, respectively. The lowest electrostatic potential on sulfur is $-32 \mathrm{~kJ} \mathrm{~mol}^{-1}$ in EDOT- $2 A$ and the values of each EDOT are $-79,-65$, and $-48 \mathrm{~kJ} \mathrm{~mol}^{-1}$ for EDOT (calculated), EDOT-1, and EDOT-3, respectively. These results show the outflowing of the surface electrons due to the formation of the co-crystal and the corresponding intermolecular interactions.

\section{Synthesis}

To a solution of $\mathrm{Cu}(L)_{2}(15 \mathrm{mg}, 17 \mu \mathrm{mol})$ in chloroform $(2 \mathrm{ml})$ was added an excess amount of EDOT. The solution was evaporated slowly to give green crystals of $\mathrm{Cu}(L)_{2} \cdot 3$ EDOT (I), which were separated by filtration and characterized by crystallographic and thermogravimetric (TG) analyses.

\section{Thermogravimetric studies}

In the TG analysis for (I), the weight loss indicates an approximate one-step elimination (Fig. 6); the total elimination of EDOT was found to be $33.6 \%$, which is almost the same as the calculated value of $33.0 \%$ around $50-130^{\circ} \mathrm{C}$. The release curve is gentle, and the coordinated EDOT and solvated EDOT are gradually separated from the crystals without being distinguished, confirming the weak coordination bond due to the Jahn-Teller effect of the $\mathrm{Cu}$ ion. In the complex, the positive electrostatic potential on the copper $\left(+206.41 \mathrm{~kJ} \mathrm{~mol}^{-1}\right)$ in the independent crystal of $\mathrm{Cu}(L)_{2}$ was higher than that of the corresponding non-fluorinated complex, $+116.71 \mathrm{~kJ} \mathrm{~mol}^{-1}$ for $\mathrm{Cu}(\mathrm{dbm})_{2}$ (Kusakawa et al., 2020) due to the substitution of the pentafluorophenyl groups, indicating that the present EDOT recognition was induced. For the same procedure, $\operatorname{Pd}(L)_{2}$ and EDOT were combined to

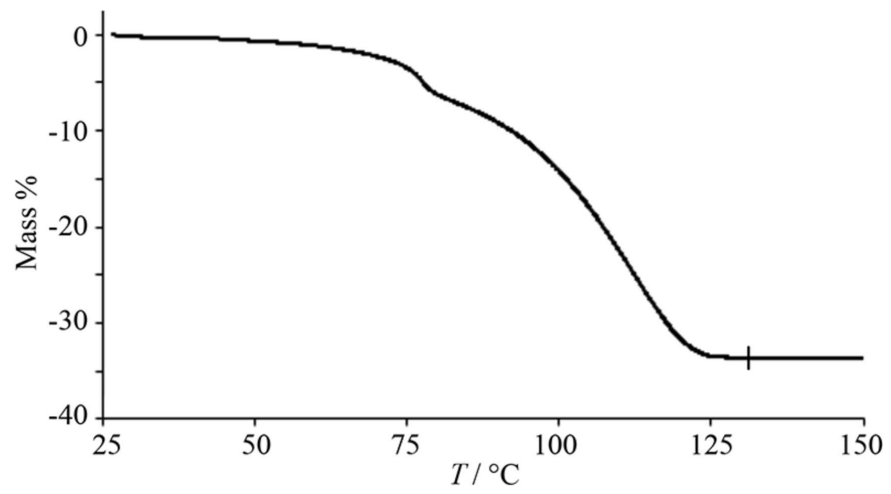

Figure 6

TG curves of (I) showing the one-step elimination; the scan rate was $5.0^{\circ} \mathrm{C} \mathrm{min}^{-1}$.
Table 2

Experimental details.

\begin{tabular}{|c|c|}
\hline \multicolumn{2}{|l|}{ Crystal data } \\
\hline Chemical formula & {$\left[\mathrm{Cu}\left(\mathrm{C}_{15} \mathrm{HF}_{10} \mathrm{O}_{2}\right)_{2}\right] \cdot 3\left(\mathrm{C}_{6} \mathrm{H}_{6} \mathrm{O}_{2} \mathrm{~S}\right)$} \\
\hline$M_{\mathrm{r}}$ & 1296.36 \\
\hline Crystal system, space group & Monoclinic, $P 2_{1} / c$ \\
\hline Temperature $(\mathrm{K})$ & 100 \\
\hline$a, b, c(\AA)$ & $\begin{array}{l}7.7343(3), 46.8973(16) \\
13.2580(5)\end{array}$ \\
\hline$\beta\left({ }^{\circ}\right)$ & $99.211(1)$ \\
\hline$V\left(\AA^{6}\right)$ & $4746.9(3)$ \\
\hline$Z$ & 4 \\
\hline Radiation type & Мo $K \alpha$ \\
\hline$\mu\left(\mathrm{mm}^{-1}\right)$ & 0.73 \\
\hline Crystal size $(\mathrm{mm})$ & $0.17 \times 0.17 \times 0.11$ \\
\hline \multicolumn{2}{|l|}{ Data collection } \\
\hline Diffractometer & Bruker D8 Goniometer \\
\hline Absorption correction & $\begin{array}{l}\text { Multi-scan (SADABS; Bruker, } \\
\text { 2018) }\end{array}$ \\
\hline$T_{\min }, T_{\max }$ & $0.88,0.93$ \\
\hline $\begin{array}{l}\text { No. of measured, independent and } \\
\text { observed }[I>2 \sigma(I)] \text { reflections }\end{array}$ & $54634,8367,7663$ \\
\hline$R_{\text {int }}$ & 0.042 \\
\hline$(\sin \theta / \lambda)_{\max }\left(\AA^{-1}\right)$ & 0.595 \\
\hline \multicolumn{2}{|l|}{ Refinement } \\
\hline$R\left[F^{2}>2 \sigma\left(F^{2}\right)\right], w R\left(F^{2}\right), S$ & $0.049,0.106,1.06$ \\
\hline No. of reflections & 8367 \\
\hline No. of parameters & 821 \\
\hline No. of restraints & 236 \\
\hline $\mathrm{H}$-atom treatment & $\mathrm{H}$-atom parameters constrained \\
\hline$\Delta \rho_{\max }, \Delta \rho_{\min }\left(\mathrm{e} \AA^{-3}\right)$ & $1.74,-1.69$ \\
\hline
\end{tabular}

Computer programs: APEX3 (Bruker, 2018), SAINT (Bruker, 2018), SHELXT2014/5 (Sheldrick, 2015a), SHELXL2018/3 (Sheldrick, 2015b) and shelXle (Hübschle et al., 2011)

give brown needle-shaped crystals, which are clearly characterized as $\operatorname{Pd}(L)_{2}$ as a single component (Nakajima \& Hori, 2014) and no guest release was observed by the brown crystals of $\operatorname{Pd}(L)_{2}$; the electrostatic potentials on the metal center of $\operatorname{Pd}(L)_{2}$ and $\operatorname{Pd}(\mathrm{dbm})_{2}$ are -1.0 and $-73 \mathrm{~kJ} \mathrm{~mol}^{-1}$, respectively (Kusakawa et al., 2020).

In summary, we have discussed the crystal structure and the intermolecular interactions for three EDOT molecules inserted in (I), in which guest recognition is induced by the flexible orientations and positive electrostatic potentials of the pentafluorophenyl groups and the enhanced positive potential on the copper ion of the fluorinated complex, $\mathrm{Cu}(L)_{2}$. The crystal structure clearly suggests that the alternate coordination polymer between the metal center of $\mathrm{Cu}(L)_{2}$ and the oxygen atom of EDOT-1 was obtained along the $a$ axis through the weak coordination bond and the close stacking between the pentafluorophenyl group of $\mathrm{Cu}(L)_{2}$ and the aromatic moiety of EDOT-2 and EDOT-3 was obtained through the arene-perfluoroarene interactions.

\section{Refinement}

Crystal data, data collection and structure refinement details are summarized in Table $2 . \mathrm{H}$ atoms were placed in geometrically idealized positions and refined as riding with $\mathrm{C}-\mathrm{H}=$ $0.95 \AA$ and $U_{\text {iso }}(\mathrm{H})=1.2 U_{\text {eq }}(\mathrm{C})$ for aromatic. 


\section{Funding information}

Funding for this research was provided by: JSPS KAKENHI (grant No. 18 K05153).

\section{References}

Bruker (2018). APEX3, SAINT, SADABS . Bruker AXS Inc., Madison, Wisconsin, USA.

Crowder, J. M., Han, H., Wei, Z., Dikarev, E. V. \& Petrukhina, M. A. (2019). Polyhedron, 157, 33-38.

Groenendaal, L., Jonas, F., Freitag, D., Pielartzik, H. \& Reynolds, J. R. (2000). Adv. Mater. 12, 481-494.

Hirshfeld, F. L. (1977). Theor. Chim. Acta, 44, 129-138.

Hori, A. (2012). Frontiers in Crystal Engineering, Vol. III, pp. 163185. New York: John Wiley \& Sons.

Hori, A. \& Arii, T. (2007). CrystEngComm, 9, 215-217.

Hori, A., Gonda, R. \& Rzeznicka, I. I. (2017). CrystEngComm, 19, 6263-6266.

Hori, A., Nakajima, K., Akimoto, Y., Naganuma, K. \& Yuge, H. (2014). CrystEngComm, 16, 8805-8817.

Hübschle, C. B., Sheldrick, G. M. \& Dittrich, B. (2011). J. Appl. Cryst. 44, 1281-1284.
Hunter, C. A. (1994). Chem. Soc. Rev. 23, 101-109.

Kirchmeyer, S. \& Reuter, K. (2005). J. Mater. Chem. 15, 2077-2088.

Kusakawa, T., Goto, T. \& Hori, A. (2020). CrystEngComm. In the press. doi: https://doi.org/10.1039/D0CE00416B.

Ma, J. C. \& Dougherty, D. A. (1997). Chem. Rev. 97, 1303-1324.

McKinnon, J. J., Jayatilaka, D. \& Spackman, M. A. (2007). Chem. Commun. pp. 3814-3816.

Nakajima, K. \& Hori, A. (2014). Cryst. Growth Des. 14, 3169-3173.

Sheldrick, G. M. (2015a). Acta Cryst. A71, 3-8.

Sheldrick, G. M. (2015b). Acta Cryst. C71, 3-8.

Skotheim, T. A., Elsenbaumer, R. L. \& Reynolds, J. R. (1998). Handbook of Conducting Polymers, 2nd ed. New York: Marcel Dekker.

Spackman, M. A. \& Jayatilaka, D. (2009). CrystEngComm, 11, 1932.

Storsberg, J., Ritter, H., Pielartzik, H. \& Groenendaal, L. (2000). Adv. Mater. 12, 567-569.

Turner, M. J., McKinnon, J. J., Wolff, S. K., Grimwood, D. J., Spackman, P. R., Jayatilaka, D. \& Spackman, M. A. (2017). Crystal Explorer 17. The University of Western Australia.

Williams, J. H. (1993). Acc. Chem. Res. 26, 593-598.

Williams, J. H. (2017). Crystal Engineering: How Molecules Build Solids. San Rafael: Morgan \& Claypool Publishers. 


\section{supporting information}

Acta Cryst. (2020). E76, 820-825 [https://doi.org/10.1107/S2056989020006155]

Co-crystal structure, Hirshfeld surface analysis and DFT studies of 3,4-ethylenedioxythiophene solvated bis[1,3-bis(pentafluorophenyl)propane-1,3dionato]copper(II)

Yusuke Habuka, Emily Ami Takeuchi and Akiko Hori

Computing details

Data collection: APEX3 (Bruker, 2018); cell refinement: APEX3 (Bruker, 2018); data reduction: SAINT (Bruker, 2018); program(s) used to solve structure: SHELXT2014/5 (Sheldrick, 2015a); program(s) used to refine structure:

SHELXL2018/3 (Sheldrick, 2015b); molecular graphics: shelXle (Hübschle et al., 2011).

Bis[1,3-bis(pentafluorophenyl) propane-1,3-dionato] copper(II) 3,4-ethylenedioxythiophene trisolvate

\section{Crystal data}

$\left[\mathrm{Cu}\left(\mathrm{C}_{15} \mathrm{HF}_{10} \mathrm{O}_{2}\right)_{2}\right] \cdot 3\left(\mathrm{C}_{6} \mathrm{H}_{6} \mathrm{O}_{2} \mathrm{~S}\right)$

$M_{r}=1296.36$

Monoclinic, $P 2_{1} / c$

$a=7.7343(3) \AA$

$b=46.8973(16) \AA$

$c=13.2580(5) \AA$

$\beta=99.211(1)^{\circ}$

$V=4746.9(3) \AA^{3}$

$Z=4$

\section{Data collection}

Bruker D8 Goniometer diffractometer

Detector resolution: 7.3910 pixels $\mathrm{mm}^{-1}$ $\varphi$ and $\omega$ scans

Absorption correction: multi-scan

(SADABS; Bruker, 2018)

$T_{\text {min }}=0.88, T_{\text {max }}=0.93$

54634 measured reflections

\section{Refinement}

Refinement on $F^{2}$

Least-squares matrix: full

$R\left[F^{2}>2 \sigma\left(F^{2}\right)\right]=0.049$

$w R\left(F^{2}\right)=0.106$

$S=1.06$

8367 reflections

821 parameters

236 restraints
$F(000)=2580$

$D_{\mathrm{x}}=1.814 \mathrm{Mg} \mathrm{m}^{-3}$

Mo $K \alpha$ radiation, $\lambda=0.71073 \AA$

Cell parameters from 9808 reflections

$\theta=2.6-26.4^{\circ}$

$\mu=0.73 \mathrm{~mm}^{-1}$

$T=100 \mathrm{~K}$

Prismatic, green

$0.17 \times 0.17 \times 0.11 \mathrm{~mm}$

8367 independent reflections

7663 reflections with $I>2 \sigma(I)$

$R_{\text {int }}=0.042$

$\theta_{\text {max }}=25.0^{\circ}, \theta_{\text {min }}=2.3^{\circ}$

$h=-9 \rightarrow 9$

$k=-55 \rightarrow 55$

$l=-15 \rightarrow 15$

Hydrogen site location: inferred from neighbouring sites

$\mathrm{H}$-atom parameters constrained

$w=1 /\left[\sigma^{2}\left(F_{\mathrm{o}}{ }^{2}\right)+(0.0217 P)^{2}+17.5257 P\right]$

where $P=\left(F_{\mathrm{o}}{ }^{2}+2 F_{\mathrm{c}}{ }^{2}\right) / 3$

$(\Delta / \sigma)_{\max }=0.001$

$\Delta \rho_{\max }=1.74 \mathrm{e} \AA^{-3}$

$\Delta \rho_{\min }=-1.69$ e $\AA^{-3}$ 


\section{Special details}

Geometry. All esds (except the esd in the dihedral angle between two 1.s. planes) are estimated using the full covariance matrix. The cell esds are taken into account individually in the estimation of esds in distances, angles and torsion angles; correlations between esds in cell parameters are only used when they are defined by crystal symmetry. An approximate (isotropic) treatment of cell esds is used for estimating esds involving l.s. planes.

Fractional atomic coordinates and isotropic or equivalent isotropic displacement parameters $\left(\AA^{2}\right)$

\begin{tabular}{|c|c|c|c|c|c|}
\hline & $x$ & $y$ & $z$ & $U_{\text {iso }} * / U_{\text {eq }}$ & Occ. $(<1)$ \\
\hline $\mathrm{C} 1$ & $0.7594(4)$ & $0.57893(7)$ & 0.7738 & $0.0197(7)$ & \\
\hline $\mathrm{C} 2$ & $0.7513(5)$ & $0.56890(7)$ & 0.8708 & $0.0239(7)$ & \\
\hline $\mathrm{C} 3$ & $0.5906(5)$ & $0.56378(7)$ & $0.8994(2)$ & $0.0230(7)$ & \\
\hline $\mathrm{C} 4$ & $0.4391(4)$ & $0.56870(7)$ & $0.8317(3)$ & $0.0202(7)$ & \\
\hline $\mathrm{C} 5$ & $0.4504(4)$ & $0.57829(6)$ & $0.7348(2)$ & $0.0161(6)$ & \\
\hline C6 & $0.6098(4)$ & $0.58382(6)$ & $0.7030(2)$ & $0.0147(6)$ & \\
\hline $\mathrm{C} 7$ & $0.6196(4)$ & $0.59398(6)$ & $0.5964(2)$ & $0.0147(6)$ & \\
\hline $\mathrm{C} 8$ & $0.5525(4)$ & $0.57585(7)$ & $0.5164(2)$ & $0.0167(6)$ & \\
\hline $\mathrm{H} 8$ & 0.504456 & 0.558107 & 0.532651 & $0.020^{*}$ & \\
\hline C9 & $0.5522(4)$ & $0.58236(6)$ & $0.4136(2)$ & $0.0161(6)$ & \\
\hline $\mathrm{C} 10$ & $0.4813(4)$ & $0.56082(7)$ & $0.3334(2)$ & $0.0181(7)$ & \\
\hline C11 & $0.3819(5)$ & $0.56923(7)$ & 0.2409 & $0.0223(7)$ & \\
\hline $\mathrm{C} 12$ & $0.3197(5)$ & $0.55012(8)$ & $0.1642(3)$ & $0.0263(8)$ & \\
\hline $\mathrm{C} 13$ & $0.3578(5)$ & $0.52165(8)$ & $0.1781(3)$ & $0.0289(8)$ & \\
\hline $\mathrm{C} 14$ & $0.4521(5)$ & $0.51230(7)$ & $0.2688(3)$ & $0.0270(8)$ & \\
\hline $\mathrm{C} 15$ & $0.5126(4)$ & $0.53170(7)$ & $0.3443(3)$ & $0.0215(7)$ & \\
\hline C16 & $0.5439(4)$ & $0.69479(7)$ & $0.1554(3)$ & $0.0211(7)$ & \\
\hline $\mathrm{C} 17$ & $0.5208(5)$ & $0.70298(8)$ & $0.0544(3)$ & $0.0244(7)$ & \\
\hline $\mathrm{C} 18$ & $0.6640(5)$ & $0.71125(8)$ & 0.0115 & $0.0265(8)$ & \\
\hline C19 & $0.8283(5)$ & $0.71115(7)$ & 0.0703 & $0.0234(7)$ & \\
\hline $\mathrm{C} 20$ & $0.8482(4)$ & $0.70269(7)$ & $0.1712(2)$ & $0.0194(7)$ & \\
\hline $\mathrm{C} 21$ & $0.7071(4)$ & $0.69421(6)$ & $0.2165(2)$ & $0.0177(7)$ & \\
\hline $\mathrm{C} 22$ & $0.7309(4)$ & $0.68348(7)$ & $0.3249(2)$ & $0.0178(7)$ & \\
\hline $\mathrm{C} 23$ & $0.8210(4)$ & $0.70030(7)$ & $0.4022(2)$ & $0.0201(7)$ & \\
\hline $\mathrm{H} 23$ & 0.868645 & 0.717911 & 0.384186 & $0.024^{*}$ & \\
\hline $\mathrm{C} 24$ & $0.8448(4)$ & $0.69249(7)$ & $0.5051(2)$ & $0.0173(7)$ & \\
\hline $\mathrm{C} 25$ & $0.9650(4)$ & $0.71009(6)$ & $0.5807(2)$ & $0.0171(7)$ & \\
\hline $\mathrm{C} 26$ & $0.9238(4)$ & $0.71685(7)$ & $0.6765(3)$ & $0.0203(7)$ & \\
\hline $\mathrm{C} 27$ & $1.0395(5)$ & $0.73123(7)$ & $0.7492(2)$ & $0.0217(7)$ & \\
\hline $\mathrm{C} 28$ & $1.2028(5)$ & $0.73887(7)$ & 0.7288 & $0.0242(8)$ & \\
\hline C29 & $1.2486(4)$ & $0.73251(7)$ & 0.6353 & $0.0215(7)$ & \\
\hline $\mathrm{C} 30$ & $1.1306(4)$ & $0.71850(7)$ & $0.5624(2)$ & $0.0185(7)$ & \\
\hline $\mathrm{Cu} 1$ & $0.68190(5)$ & $0.63872(2)$ & $0.46222(3)$ & $0.01411(10)$ & \\
\hline $\mathrm{F} 1$ & $0.9178(2)$ & $0.58323(5)$ & $0.74715(16)$ & $0.0310(5)$ & \\
\hline $\mathrm{F} 2$ & $0.8979(3)$ & $0.56388(5)$ & $0.93711(16)$ & $0.0374(5)$ & \\
\hline F3 & $0.5815(3)$ & $0.55400(5)$ & $0.99358(14)$ & $0.0313(5)$ & \\
\hline F4 & $0.2828(3)$ & $0.56383(5)$ & $0.85960(15)$ & $0.0323(5)$ & \\
\hline F5 & $0.3000(2)$ & $0.58185(4)$ & $0.66859(14)$ & $0.0241(4)$ & \\
\hline F6 & $0.3354(3)$ & $0.59659(4)$ & $0.22499(15)$ & $0.0325(5)$ & \\
\hline
\end{tabular}




\begin{tabular}{|c|c|c|c|c|c|}
\hline F7 & $0.2237(3)$ & $0.55922(5)$ & $0.07760(15)$ & $0.0357(5)$ & \\
\hline F8 & $0.3024(3)$ & $0.50278(5)$ & $0.10381(17)$ & $0.0414(6)$ & \\
\hline F9 & $0.4876(3)$ & $0.48451(4)$ & $0.28398(18)$ & $0.0411(6)$ & \\
\hline F10 & $0.6066(3)$ & $0.52110(4)$ & $0.43069(15)$ & $0.0271(5)$ & \\
\hline F11 & $0.4006(3)$ & $0.68757(5)$ & $0.19509(15)$ & $0.0315(5)$ & \\
\hline F12 & $0.3608(3)$ & $0.70347(5)$ & $-0.00156(16)$ & $0.0382(5)$ & \\
\hline F13 & $0.6433(3)$ & $0.71930(6)$ & $-0.08624(16)$ & $0.0436(6)$ & \\
\hline F14 & $0.9691(3)$ & $0.71899(5)$ & $0.02976(16)$ & $0.0380(6)$ & \\
\hline F15 & $1.0123(2)$ & $0.70194(4)$ & $0.22413(14)$ & $0.0262(4)$ & \\
\hline F16 & $0.7661(3)$ & $0.71015(4)$ & $0.69951(15)$ & $0.0281(5)$ & \\
\hline F17 & $0.9945(3)$ & $0.73766(4)$ & $0.83999(15)$ & $0.0310(5)$ & \\
\hline F18 & $1.3151(3)$ & $0.75249(5)$ & $0.79938(16)$ & $0.0344(5)$ & \\
\hline F19 & $1.4079(3)$ & $0.73923(5)$ & $0.61553(17)$ & $0.0317(5)$ & \\
\hline F20 & $1.1831(2)$ & $0.71248(4)$ & $0.47335(14)$ & $0.0232(4)$ & \\
\hline $\mathrm{O} 1$ & $0.6911(3)$ & $0.61812(4)$ & $0.58985(16)$ & $0.0160(5)$ & \\
\hline $\mathrm{O} 2$ & $0.6045(3)$ & $0.60555(5)$ & $0.37963(16)$ & $0.0174(5)$ & \\
\hline $\mathrm{O} 3$ & $0.6659(3)$ & $0.65885(5)$ & $0.33475(16)$ & $0.0183(5)$ & \\
\hline $\mathrm{O} 4$ & $0.7762(3)$ & $0.67114(4)$ & $0.54204(16)$ & $0.0161(5)$ & \\
\hline $\mathrm{C} 31$ & $0.1008(4)$ & $0.64236(7)$ & $0.6576(3)$ & $0.0206(7)$ & \\
\hline H31 & -0.006458 & 0.635978 & 0.676158 & $0.025^{*}$ & \\
\hline $\mathrm{C} 32$ & $0.1423(4)$ & $0.64075(7)$ & $0.5623(2)$ & $0.0175(6)$ & \\
\hline $\mathrm{C} 33$ & $0.3118(4)$ & $0.65181(7)$ & $0.5562(2)$ & $0.0174(7)$ & \\
\hline C34 & $0.3973(4)$ & $0.66157(7)$ & $0.6465(3)$ & $0.0217(7)$ & \\
\hline H34 & 0.511792 & 0.669515 & 0.656735 & $0.026^{*}$ & \\
\hline $\mathrm{C} 35$ & $0.1249(4)$ & $0.62454(7)$ & $0.3939(3)$ & $0.0224(7)$ & \\
\hline $\mathrm{H} 35 \mathrm{~A}$ & 0.039407 & 0.621591 & 0.330723 & $0.027^{*}$ & \\
\hline H35B & 0.194058 & 0.606808 & 0.407871 & $0.027^{*}$ & \\
\hline $\mathrm{C} 36$ & $0.2450(4)$ & $0.64868(7)$ & $0.3781(2)$ & $0.0203(7)$ & \\
\hline H36A & 0.299577 & 0.644895 & 0.316742 & $0.024^{*}$ & \\
\hline H36B & 0.176752 & 0.666565 & 0.366393 & $0.024^{*}$ & \\
\hline O5 & $0.0319(3)$ & $0.63026(5)$ & $0.47813(17)$ & $0.0219(5)$ & \\
\hline O6 & $0.3806(3)$ & $0.65200(5)$ & $0.46638(17)$ & $0.0196(5)$ & \\
\hline S1 & $0.26923(11)$ & $0.65754(2)$ & $0.74025(6)$ & $0.02224(19)$ & \\
\hline C37A & $0.6149(13)$ & $0.63435(13)$ & $-0.0633(7)$ & $0.081(2)$ & $0.691(4)$ \\
\hline H37A & 0.563149 & 0.637854 & -0.132152 & $0.097^{*}$ & $0.691(4)$ \\
\hline C38A & $0.8040(15)$ & $0.63579(9)$ & $-0.0290(9)$ & $0.065(2)$ & $0.691(4)$ \\
\hline C39A & $0.8335(12)$ & $0.63070(10)$ & $0.0769(6)$ & $0.0505(18)$ & $0.691(4)$ \\
\hline C40A & $0.6864(13)$ & $0.62610(15)$ & $0.1133(9)$ & $0.070(2)$ & $0.691(4)$ \\
\hline $\mathrm{H} 40 \mathrm{~A}$ & 0.682749 & 0.622829 & 0.183669 & $0.084^{*}$ & $0.691(4)$ \\
\hline $\mathrm{C} 41 \mathrm{~A}$ & $1.1061(13)$ & $0.6324(2)$ & $-0.0266(6)$ & $0.076(2)$ & $0.691(4)$ \\
\hline $\mathrm{H} 41 \mathrm{~A}$ & 1.200067 & 0.640480 & -0.060666 & $0.091^{*}$ & $0.691(4)$ \\
\hline H41B & 1.116849 & 0.611377 & -0.026951 & $0.091 *$ & $0.691(4)$ \\
\hline $\mathrm{C} 42 \mathrm{~A}$ & $1.1269(11)$ & $0.64274(16)$ & $0.0798(5)$ & $0.0560(18)$ & $0.691(4)$ \\
\hline $\mathrm{H} 42 \mathrm{~A}$ & 1.245676 & 0.637829 & 0.115366 & $0.067^{*}$ & $0.691(4)$ \\
\hline H42B & 1.115506 & 0.663764 & 0.079751 & $0.067^{*}$ & $0.691(4)$ \\
\hline O7A & $0.9340(10)$ & $0.64083(11)$ & $-0.0833(4)$ & $0.0793(18)$ & $0.691(4)$ \\
\hline O8A & $1.0018(9)$ & $0.63079(13)$ & $0.1331(6)$ & $0.0464(17)$ & $0.691(4)$ \\
\hline S2A & $0.5036(4)$ & $0.62657(5)$ & $0.0220(3)$ & $0.0935(11)$ & $0.691(4)$ \\
\hline
\end{tabular}




\begin{tabular}{|c|c|c|c|c|c|}
\hline C37B & $0.7270(17)$ & $0.6447(2)$ & $-0.1070(9)$ & $0.026(2)$ & 0.309 (4) \\
\hline H37B & 0.629116 & 0.642764 & -0.159856 & $0.032 *$ & $0.309(4)$ \\
\hline C38B & $0.741(2)$ & $0.6349(2)$ & $-0.0184(14)$ & $0.027(3)$ & 0.309 (4) \\
\hline C39B & $0.9021(17)$ & $0.6392(2)$ & $0.0503(10)$ & $0.026(2)$ & $0.309(4)$ \\
\hline C40B & 1.0267 (17) & $0.6549(2)$ & $0.0030(10)$ & $0.033(3)$ & $0.309(4)$ \\
\hline $\mathrm{H} 40 \mathrm{~B}$ & 1.142956 & 0.659473 & 0.033626 & $0.040^{*}$ & 0.309 (4) \\
\hline C41B & $0.644(2)$ & $0.6146(4)$ & $0.1222(13)$ & $0.044(3)$ & 0.309 (4) \\
\hline $\mathrm{H} 41 \mathrm{C}$ & 0.619068 & 0.631998 & 0.159730 & $0.053 *$ & 0.309 (4) \\
\hline $\mathrm{H} 41 \mathrm{D}$ & 0.562812 & 0.599481 & 0.138370 & $0.053^{*}$ & 0.309 (4) \\
\hline C42B & $0.823(2)$ & $0.6056(3)$ & 0.1583 (11) & $0.048(3)$ & $0.309(4)$ \\
\hline $\mathrm{H} 42 \mathrm{C}$ & 0.854016 & 0.589377 & 0.116939 & $0.058 *$ & $0.309(4)$ \\
\hline $\mathrm{H} 42 \mathrm{D}$ & 0.835506 & 0.599434 & 0.230543 & $0.058^{*}$ & 0.309 (4) \\
\hline O7B & $0.6071(13)$ & $0.6205(2)$ & $0.0122(8)$ & $0.043(2)$ & 0.309 (4) \\
\hline O8B & 0.9395 (18) & $0.6302(3)$ & $0.1485(12)$ & $0.035(3)$ & 0.309 (4) \\
\hline S2B & $0.9318(6)$ & $0.66369(8)$ & $-0.1156(3)$ & $0.0442(12)$ & 0.309 (4) \\
\hline $\mathrm{C} 43$ & $0.0201(7)$ & $0.50447(11)$ & $0.7530(4)$ & $0.0524(12)$ & \\
\hline H43 & -0.018735 & 0.523709 & 0.751083 & $0.063^{*}$ & \\
\hline C44 & $-0.0114(5)$ & $0.48559(9)$ & $0.6737(3)$ & $0.0328(9)$ & \\
\hline $\mathrm{C} 45$ & $0.0687(5)$ & $0.45912(10)$ & $0.6969(3)$ & $0.0356(9)$ & \\
\hline C46 & $0.1565(7)$ & $0.45771(13)$ & $0.7943(3)$ & $0.0626(16)$ & \\
\hline H46 & 0.218746 & 0.441339 & 0.822400 & $0.075^{*}$ & \\
\hline C47 & $-0.1409(5)$ & $0.46667(9)$ & $0.5206(3)$ & $0.0311(9)$ & \\
\hline H47A & -0.179232 & 0.472340 & 0.448601 & $0.037^{*}$ & \\
\hline H47B & -0.239788 & 0.456524 & 0.543732 & $0.037^{*}$ & \\
\hline $\mathrm{C} 48$ & $0.0045(6)$ & $0.44703(8)$ & $0.5246(3)$ & $0.0366(9)$ & \\
\hline $\mathrm{H} 48 \mathrm{~A}$ & -0.031048 & 0.430597 & 0.479103 & $0.044^{*}$ & \\
\hline $\mathrm{H} 48 \mathrm{~B}$ & 0.103712 & 0.456699 & 0.500110 & $0.044 *$ & \\
\hline O9 & $-0.1054(4)$ & $0.49163(6)$ & $0.5800(2)$ & $0.0412(7)$ & \\
\hline $\mathrm{O} 10$ & $0.0591(4)$ & $0.43703(6)$ & $0.6276(2)$ & $0.0443(7)$ & \\
\hline $\mathrm{S} 3$ & $0.14290(18)$ & $0.48766(4)$ & $0.85819(10)$ & $0.0653(4)$ & \\
\hline
\end{tabular}

Atomic displacement parameters $\left(\AA^{2}\right)$

\begin{tabular}{lllllll}
\hline & $U^{11}$ & $U^{22}$ & $U^{33}$ & $U^{12}$ & $U^{13}$ & $U^{23}$ \\
\hline C1 & $0.0169(16)$ & $0.0194(16)$ & $0.0221(17)$ & $-0.0026(13)$ & $0.0010(13)$ & $0.0019(13)$ \\
C2 & $0.0243(18)$ & $0.0256(18)$ & $0.0186(17)$ & $-0.0002(14)$ & $-0.0061(14)$ & $0.0014(14)$ \\
C3 & $0.035(2)$ & $0.0201(17)$ & $0.0139(16)$ & $-0.0022(15)$ & $0.0036(14)$ & $-0.0006(13)$ \\
C4 & $0.0212(17)$ & $0.0202(17)$ & $0.0207(17)$ & $-0.0016(13)$ & $0.0079(13)$ & $-0.0019(13)$ \\
C5 & $0.0164(16)$ & $0.0140(15)$ & $0.0167(16)$ & $0.0006(12)$ & $-0.0011(12)$ & $-0.0003(12)$ \\
C6 & $0.0187(16)$ & $0.0098(14)$ & $0.0154(15)$ & $-0.0015(12)$ & $0.0020(12)$ & $-0.0012(12)$ \\
C7 & $0.0105(14)$ & $0.0151(15)$ & $0.0182(16)$ & $0.0031(12)$ & $0.0016(12)$ & $0.0011(12)$ \\
C8 & $0.0193(16)$ & $0.0120(15)$ & $0.0184(16)$ & $-0.0036(12)$ & $0.0022(13)$ & $-0.0003(12)$ \\
C9 & $0.0137(15)$ & $0.0145(16)$ & $0.0197(16)$ & $0.0020(12)$ & $0.0008(12)$ & $-0.0023(13)$ \\
C10 & $0.0191(16)$ & $0.0183(16)$ & $0.0167(16)$ & $-0.0003(13)$ & $0.0025(13)$ & $-0.0035(13)$ \\
C11 & $0.0261(18)$ & $0.0197(17)$ & $0.0204(17)$ & $0.0034(14)$ & $0.0019(14)$ & $-0.0033(14)$ \\
C12 & $0.0258(19)$ & $0.034(2)$ & $0.0176(17)$ & $0.0021(15)$ & $-0.0022(14)$ & $-0.0024(15)$ \\
C13 & $0.032(2)$ & $0.028(2)$ & $0.0238(18)$ & $-0.0012(16)$ & $-0.0025(15)$ & $-0.0154(15)$ \\
C14 & $0.032(2)$ & $0.0165(17)$ & $0.031(2)$ & $0.0015(14)$ & $0.0003(16)$ & $-0.0069(15)$
\end{tabular}




\begin{tabular}{|c|c|c|c|c|c|c|}
\hline $\mathrm{C} 15$ & $0.0210(17)$ & $0.0215(17)$ & $0.0201(17)$ & $0.0011(14)$ & $-0.0025(13)$ & $-0.0037(14)$ \\
\hline $\mathrm{C} 16$ & $0.0208(17)$ & $0.0191(17)$ & $0.0236(17)$ & $-0.0019(13)$ & $0.0040(14)$ & $0.0001(14)$ \\
\hline $\mathrm{C} 17$ & $0.0236(18)$ & $0.0260(18)$ & $0.0217(17)$ & $0.0009(14)$ & -0.0023 & $0.0026(14)$ \\
\hline $\mathrm{C} 18$ & $0.036(2)$ & $0.0291(19)$ & $0.0136(16)$ & $0.0021(16)$ & $0.0011(15)$ & $0.0077(14)$ \\
\hline C19 & $0.0261(18)$ & $0.0240(18)$ & $0.0215(17)$ & $-0.0026(14)$ & $0.0078(14)$ & $0.0052(14)$ \\
\hline $\mathrm{C} 20$ & $0.0217(17)$ & $0.0149(16)$ & $0.0204(16)$ & $-0.0024(13)$ & $0.0000(13)$ & $0.0005(13)$ \\
\hline $\mathrm{C} 21$ & $0.0250(17)$ & $0.0112(15)$ & $0.0172(16)$ & -0.0025 & $0.0038(13)$ & $0.0002(12)$ \\
\hline $\mathrm{C} 22$ & $0.0173(16)$ & $0.0178(16)$ & $0.0188(16)$ & $-0.0008(13)$ & $0.0044(13)$ & $0.0012(13)$ \\
\hline $\mathrm{C} 23$ & $0.0242(17)$ & $0.0163(16)$ & $0.0193(16)$ & $-0.0065(13)$ & $0.0023(13)$ & $0.0008(13)$ \\
\hline $\mathrm{C} 24$ & $0.0163(16)$ & $0.0138(15)$ & $0.0226(17)$ & $0.0005(12)$ & $0.0058(13)$ & $-0.0029(13)$ \\
\hline $\mathrm{C} 25$ & $0.0213(17)$ & $0.0112(15)$ & $0.0190(16)$ & $-0.0029(12)$ & $0.0039(13)$ & $0.0016(12)$ \\
\hline $\mathrm{C} 26$ & $0.0209(17)$ & $0.0166(16)$ & $0.0243(17)$ & $0.0003(13)$ & $0.0060(14)$ & $-0.0007(13)$ \\
\hline $\mathrm{C} 27$ & $0.033(2)$ & $0.0153(16)$ & $0.0167(16)$ & $0.0019(14)$ & $0.0044(14)$ & $-0.0025(13)$ \\
\hline $\mathrm{C} 28$ & $0.0309(19)$ & $0.0133(16)$ & $0.0254(18)$ & $-0.0023(14)$ & $-0.0047(15)$ & $-0.0032(14)$ \\
\hline $\mathrm{C} 29$ & $0.0193(17)$ & $0.0165(16)$ & $0.0287(18)$ & $-0.0025(13)$ & $0.0033(14)$ & $0.0006(14)$ \\
\hline $\mathrm{C} 30$ & $0.0238(17)$ & $0.0137(15)$ & $0.0187(16)$ & $0.0000(13)$ & $0.0058(13)$ & $-0.0002(13)$ \\
\hline $\mathrm{Cu} 1$ & $0.0160(2)$ & 0.01277 (19) & $0.01372(19)$ & $-0.00238(14)$ & $0.00286(14)$ & -0.00015 \\
\hline F1 & $0.0151(10)$ & $0.0467(13)$ & $0.0298(11)$ & $-0.0041(9)$ & $-0.0011(8)$ & $0.0100(10)$ \\
\hline $\mathrm{F} 2$ & $0.0266(11)$ & $0.0555(15)$ & $0.0250(11)$ & $0.0015(10)$ & $-0.0108(9)$ & $0.0114(10)$ \\
\hline F3 & $0.0435(13)$ & $0.0369(12)$ & $0.0134(9)$ & $0.0006(10)$ & $0.0042(9)$ & $0.0057(9)$ \\
\hline $\mathrm{F} 4$ & $0.0260(11)$ & $0.0471(13)$ & $0.0263(11)$ & $-0.0014(10)$ & $0.0118(9)$ & $0.0063(10)$ \\
\hline F5 & $0.0156(9)$ & $0.0345(11)$ & $0.0211(10)$ & $0.0014(8)$ & $-0.0002(8)$ & $0.0049(8)$ \\
\hline F6 & $0.0462(13)$ & $0.0219(11)$ & $0.0243(11)$ & $0.0109(9)$ & $-0.0097(9)$ & $-0.0028(8)$ \\
\hline F7 & $0.0414(13)$ & $0.0422(13)$ & $0.0187(10)$ & $0.0043(10)$ & $-0.0100(9)$ & $-0.0043(9)$ \\
\hline F8 & $0.0543(15)$ & $0.0360(13)$ & $0.0294(12)$ & $-0.0026(11)$ & $-0.0068(11)$ & $-0.0208(10)$ \\
\hline F9 & $0.0602(16)$ & $0.0167(11)$ & $0.0412(13)$ & $0.0042(10)$ & $-0.0074(11)$ & $-0.0109(10)$ \\
\hline F10 & $0.0333(11)$ & $0.0185(10)$ & $0.0253(10)$ & $0.0031(8)$ & $-0.0076(9)$ & $-0.0014(8)$ \\
\hline F11 & $0.0209(10)$ & $0.0467(13)$ & $0.0270(11)$ & $-0.0044(9)$ & $0.0046(9)$ & $0.0059(10)$ \\
\hline F12 & $0.0258(11)$ & $0.0569(15)$ & $0.0278(11)$ & $-0.0003(10)$ & $-0.0077(9)$ & $0.0093(11)$ \\
\hline F13 & $0.0419(14)$ & $0.0681(17)$ & $0.0195(11)$ & $-0.0015(12)$ & $0.0010(10)$ & $0.0185(11)$ \\
\hline F14 & $0.0329(12)$ & $0.0576(15)$ & $0.0250(11)$ & $-0.0105(11)$ & $0.0098(9)$ & $0.0122(10)$ \\
\hline F15 & $0.0215(10)$ & $0.0346(11)$ & $0.0217(10)$ & $-0.0065(9)$ & $0.0007(8)$ & $0.0056(9)$ \\
\hline F16 & $0.0272(11)$ & $0.0320(11)$ & $0.0280(11)$ & $-0.0053(9)$ & $0.0137(9)$ & $-0.0078(9)$ \\
\hline F17 & $0.0468(13)$ & $0.0271(11)$ & $0.0201(10)$ & $-0.0019(10)$ & $0.0088(9)$ & $-0.0092(9)$ \\
\hline F18 & $0.0379(13)$ & $0.0307(12)$ & $0.0301(12)$ & $-0.0077(10)$ & $-0.0080(10)$ & $-0.0086(9)$ \\
\hline F19 & $0.0208(11)$ & $0.0341(12)$ & $0.0399(13)$ & $-0.0107(9)$ & $0.0037(9)$ & $-0.0027(10)$ \\
\hline F20 & $0.0252(10)$ & $0.0256(10)$ & $0.0208(10)$ & $-0.0040(8)$ & $0.0100(8)$ & $-0.0015(8)$ \\
\hline $\mathrm{O} 1$ & $0.0170(11)$ & $0.0142(11)$ & $0.0162(11)$ & $-0.0026(9)$ & $0.0014(9)$ & $0.0004(9)$ \\
\hline $\mathrm{O} 2$ & $0.0213(12)$ & $0.0161(11)$ & $0.0150(11)$ & $-0.0010(9)$ & $0.0035(9)$ & $-0.0015(9)$ \\
\hline $\mathrm{O} 3$ & $0.0220(12)$ & $0.0165(11)$ & $0.0163(11)$ & $-0.0056(9)$ & $0.0026(9)$ & $0.0000(9)$ \\
\hline $\mathrm{O} 4$ & $0.0182(11)$ & $0.0157(11)$ & $0.0154(11)$ & $-0.0027(9)$ & $0.0051(9)$ & $-0.0010(9)$ \\
\hline $\mathrm{C} 31$ & $0.0172(16)$ & $0.0221(17)$ & $0.0230(17)$ & $0.0004(13)$ & $0.0047(13)$ & $0.0009(14)$ \\
\hline $\mathrm{C} 32$ & $0.0159(16)$ & $0.0167(16)$ & $0.0194(16)$ & $0.0023(13)$ & $0.0016(13)$ & $-0.0002(13)$ \\
\hline $\mathrm{C} 33$ & $0.0151(15)$ & $0.0193(16)$ & $0.0184(16)$ & $0.0038(13)$ & $0.0051(13)$ & $0.0018(13)$ \\
\hline $\mathrm{C} 34$ & $0.0166(16)$ & $0.0272(18)$ & $0.0217(17)$ & $0.0008(14)$ & $0.0043(13)$ & $-0.0008(14)$ \\
\hline $\mathrm{C} 35$ & $0.0213(17)$ & $0.0259(18)$ & $0.0210(17)$ & $-0.0008(14)$ & $0.0063(14)$ & $-0.0072(14)$ \\
\hline $\mathrm{C} 36$ & $0.0200(17)$ & $0.0234(17)$ & $0.0172(16)$ & $0.0025(13)$ & $0.0018(13)$ & $-0.0010(13)$ \\
\hline O5 & $0.0159(11)$ & $0.0301(13)$ & $0.0200(12)$ & $-0.0033(10)$ & $0.0037(9)$ & $-0.0073(10)$ \\
\hline
\end{tabular}




$\begin{array}{lllllll}\text { O6 } & 0.0147(11) & 0.0276(13) & 0.0172(11) & -0.0006(9) & 0.0046(9) & -0.0011(10) \\ \text { S1 } & 0.0212(4) & 0.0292(5) & 0.0160(4) & 0.0024(3) & 0.0019(3) & -0.0007(3) \\ \text { C37A } & 0.100(5) & 0.031(3) & 0.083(4) & 0.006(3) & -0.075(4) & -0.007(3) \\ \text { C38A } & 0.093(5) & 0.028(3) & 0.057(4) & -0.002(3) & -0.042(4) & -0.001(3) \\ \text { C39A } & 0.066(4) & 0.029(3) & 0.046(4) & 0.007(3) & -0.024(3) & -0.008(3) \\ \text { C40A } & 0.069(5) & 0.044(4) & 0.086(5) & 0.013(4) & -0.022(4) & -0.036(4) \\ \text { C41A } & 0.109(5) & 0.072(4) & 0.042(4) & -0.027(4) & -0.003(4) & 0.008(3) \\ \text { C42A } & 0.076(4) & 0.054(4) & 0.033(3) & -0.014(3) & -0.010(3) & 0.011(3) \\ \text { O7A } & 0.129(4) & 0.057(3) & 0.036(3) & -0.016(3) & -0.035(3) & 0.015(2) \\ \text { O8A } & 0.056(4) & 0.047(3) & 0.030(3) & -0.003(3) & -0.013(3) & 0.006(2) \\ \text { S2A } & 0.0765(18) & 0.0582(14) & 0.127(2) & 0.0261(12) & -0.0414(16) & -0.0615(15) \\ \text { C37B } & 0.033(5) & 0.028(5) & 0.017(4) & 0.013(4) & -0.001(4) & -0.006(4) \\ \text { C38B } & 0.026(5) & 0.031(5) & 0.027(5) & 0.001(4) & 0.016(4) & -0.007(4) \\ \text { C39B } & 0.027(4) & 0.027(4) & 0.024(5) & 0.004(4) & 0.006(4) & 0.000(4) \\ \text { C40B } & 0.036(5) & 0.025(5) & 0.038(5) & -0.001(4) & 0.002(4) & 0.001(4) \\ \text { C41B } & 0.050(6) & 0.054(6) & 0.033(5) & -0.014(5) & 0.022(5) & -0.009(5) \\ \text { C42B } & 0.061(6) & 0.049(5) & 0.034(5) & -0.014(5) & 0.004(5) & 0.001(5) \\ \text { O7B } & 0.025(4) & 0.064(5) & 0.048(4) & -0.018(4) & 0.033(4) & -0.028(4) \\ \text { O8B } & 0.041(6) & 0.035(5) & 0.024(5) & -0.002(4) & -0.006(5) & 0.000(4) \\ \text { S2B } & 0.070(3) & 0.031(2) & 0.0374(19) & -0.0014(16) & 0.0282(17) & 0.0000(15) \\ \text { C43 } & 0.067(3) & 0.048(3) & 0.045(3) & -0.010(2) & 0.017(2) & -0.005(2) \\ \text { C44 } & 0.031(2) & 0.036(2) & 0.030(2) & -0.0056(17) & 0.0020(16) & 0.0027(17) \\ \text { C45 } & 0.026(2) & 0.053(3) & 0.028(2) & 0.0078(18) & 0.0036(16) & 0.0055(18) \\ \text { C46 } & 0.054(3) & 0.107(5) & 0.025(2) & 0.047(3) & 0.000(2) & 0.000(2) \\ \text { C47 } & 0.0203(18) & 0.048(2) & 0.0249(19) & -0.0103(16) & 0.0036(15) & -0.0028(17) \\ \text { C48 } & 0.054(3) & 0.027(2) & 0.026(2) & -0.0040(18) & -0.0025(18) & 0.0016(16) \\ \text { O9 } & 0.0491(18) & 0.0391(16) & 0.0335(15) & 0.0116(14) & 0.0009(13) & 0.0044(13) \\ \text { O10 } & 0.058(2) & 0.0410(17) & 0.0328(16) & 0.0137(15) & 0.0037(14) & 0.0049(13) \\ \text { S3 } & 0.0512(8) & 0.1075(12) & 0.0347(6) & 0.0080(7) & -0.0008(5) & -0.0211(7)\end{array}$

Geometric parameters $\left(\AA,{ }^{\circ}\right)$

\begin{tabular}{llll}
\hline $\mathrm{C} 1-\mathrm{F} 1$ & $1.344(4)$ & $\mathrm{C} 31-\mathrm{C} 32$ & $1.355(5)$ \\
$\mathrm{C} 1-\mathrm{C} 2$ & $1.380(5)$ & $\mathrm{C} 31-\mathrm{S} 1$ & $1.717(3)$ \\
$\mathrm{C} 1-\mathrm{C} 6$ & $1.387(4)$ & $\mathrm{C} 31-\mathrm{H} 31$ & 0.9500 \\
$\mathrm{C} 2-\mathrm{F} 2$ & $1.340(4)$ & $\mathrm{C} 32-\mathrm{O} 5$ & $1.382(4)$ \\
$\mathrm{C} 2-\mathrm{C} 3$ & $1.377(5)$ & $\mathrm{C} 32-\mathrm{C} 33$ & $1.425(4)$ \\
$\mathrm{C} 3-\mathrm{F} 3$ & $1.343(4)$ & $\mathrm{C} 33-\mathrm{C} 34$ & $1.351(5)$ \\
$\mathrm{C} 3-\mathrm{C} 4$ & $1.377(5)$ & $\mathrm{C} 33-\mathrm{O} 6$ & $1.380(4)$ \\
$\mathrm{C} 4-\mathrm{F} 4$ & $1.340(4)$ & $\mathrm{C} 34-\mathrm{S} 1$ & $1.719(3)$ \\
$\mathrm{C} 4-\mathrm{C} 5$ & $1.377(5)$ & $\mathrm{C} 34-\mathrm{H} 34$ & 0.9500 \\
$\mathrm{C} 5-\mathrm{F} 5$ & $1.350(4)$ & $\mathrm{C} 35-\mathrm{O} 5$ & $1.447(4)$ \\
$\mathrm{C} 5-\mathrm{C} 6$ & $1.390(4)$ & $\mathrm{C} 35-\mathrm{C} 36$ & $1.501(5)$ \\
$\mathrm{C} 6-\mathrm{C} 7$ & $1.505(4)$ & $\mathrm{C} 35-\mathrm{H} 35 \mathrm{~A}$ & 0.9900 \\
$\mathrm{C} 7-\mathrm{O} 1$ & $1.269(4)$ & $\mathrm{C} 35-\mathrm{H} 35 \mathrm{~B}$ & 0.9900 \\
$\mathrm{C} 7-\mathrm{C} 8$ & $1.393(4)$ & $\mathrm{C} 36-\mathrm{O} 6$ & $1.450(4)$ \\
$\mathrm{C} 8-\mathrm{C} 9$ & $1.396(4)$ & $\mathrm{C} 36-\mathrm{H} 36 \mathrm{~A}$ & 0.9900 \\
$\mathrm{C} 8-\mathrm{H} 8$ & 0.9500 & $\mathrm{C} 36-\mathrm{H} 36 \mathrm{~B}$ & 0.9900
\end{tabular}




\begin{tabular}{|c|c|c|c|}
\hline $\mathrm{C} 9-\mathrm{O} 2$ & $1.268(4)$ & $\mathrm{C} 37 \mathrm{~A}-\mathrm{C} 38 \mathrm{~A}$ & $1.463(14)$ \\
\hline $\mathrm{C} 9-\mathrm{C} 10$ & $1.505(4)$ & $\mathrm{C} 37 \mathrm{~A}-\mathrm{S} 2 \mathrm{~A}$ & $1.570(11)$ \\
\hline $\mathrm{C} 10-\mathrm{C} 15$ & $1.390(5)$ & C37A-H37A & 0.9500 \\
\hline $\mathrm{C} 10-\mathrm{C} 11$ & $1.396(5)$ & $\mathrm{C} 38 \mathrm{~A}-\mathrm{O} 7 \mathrm{~A}$ & $1.347(14)$ \\
\hline $\mathrm{C} 11-\mathrm{F} 6$ & $1.340(4)$ & $\mathrm{C} 38 \mathrm{~A}-\mathrm{C} 39 \mathrm{~A}$ & 1.407 (14) \\
\hline $\mathrm{C} 11-\mathrm{C} 12$ & $1.383(5)$ & $\mathrm{C} 39 \mathrm{~A}-\mathrm{C} 40 \mathrm{~A}$ & $1.323(14)$ \\
\hline $\mathrm{C} 12-\mathrm{F} 7$ & $1.334(4)$ & $\mathrm{C} 39 \mathrm{~A}-\mathrm{O} 8 \mathrm{~A}$ & $1.392(11)$ \\
\hline $\mathrm{C} 12-\mathrm{C} 13$ & $1.373(5)$ & $\mathrm{C} 40 \mathrm{~A}-\mathrm{S} 2 \mathrm{~A}$ & $1.708(9)$ \\
\hline $\mathrm{C} 13-\mathrm{F} 8$ & $1.342(4)$ & $\mathrm{C} 40 \mathrm{~A}-\mathrm{H} 40 \mathrm{~A}$ & 0.9500 \\
\hline $\mathrm{C} 13-\mathrm{C} 14$ & $1.375(5)$ & $\mathrm{C} 41 \mathrm{~A}-\mathrm{O} 7 \mathrm{~A}$ & $1.474(11)$ \\
\hline $\mathrm{C} 14-\mathrm{F} 9$ & $1.340(4)$ & $\mathrm{C} 41 \mathrm{~A}-\mathrm{C} 42 \mathrm{~A}$ & $1.476(10)$ \\
\hline $\mathrm{C} 14-\mathrm{C} 15$ & $1.378(5)$ & $\mathrm{C} 41 \mathrm{~A}-\mathrm{H} 41 \mathrm{~A}$ & 0.9900 \\
\hline $\mathrm{C} 15-\mathrm{F} 10$ & $1.349(4)$ & $\mathrm{C} 41 \mathrm{~A}-\mathrm{H} 41 \mathrm{~B}$ & 0.9900 \\
\hline C16-F11 & $1.343(4)$ & $\mathrm{C} 42 \mathrm{~A}-\mathrm{O} 8 \mathrm{~A}$ & $1.403(10)$ \\
\hline $\mathrm{C} 16-\mathrm{C} 17$ & $1.378(5)$ & $\mathrm{C} 42 \mathrm{~A}-\mathrm{H} 42 \mathrm{~A}$ & 0.9900 \\
\hline $\mathrm{C} 16-\mathrm{C} 21$ & $1.387(5)$ & $\mathrm{C} 42 \mathrm{~A}-\mathrm{H} 42 \mathrm{~B}$ & 0.9900 \\
\hline $\mathrm{C} 17-\mathrm{F} 12$ & $1.337(4)$ & $\mathrm{C} 37 \mathrm{~B}-\mathrm{C} 38 \mathrm{~B}$ & $1.25(2)$ \\
\hline $\mathrm{C} 17-\mathrm{C} 18$ & $1.379(5)$ & $\mathrm{C} 37 \mathrm{~B}-\mathrm{S} 2 \mathrm{~B}$ & $1.836(14)$ \\
\hline C18-F13 & $1.335(4)$ & C37B-H37B & 0.9500 \\
\hline $\mathrm{C} 18-\mathrm{C} 19$ & $1.380(5)$ & $\mathrm{C} 38 \mathrm{~B}-\mathrm{O} 7 \mathrm{~B}$ & $1.351(16)$ \\
\hline C19-F14 & $1.341(4)$ & $\mathrm{C} 38 \mathrm{~B}-\mathrm{C} 39 \mathrm{~B}$ & $1.44(2)$ \\
\hline $\mathrm{C} 19-\mathrm{C} 20$ & $1.380(5)$ & $\mathrm{C} 39 \mathrm{~B}-\mathrm{O} 8 \mathrm{~B}$ & $1.36(2)$ \\
\hline $\mathrm{C} 20-\mathrm{F} 15$ & $1.348(4)$ & $\mathrm{C} 39 \mathrm{~B}-\mathrm{C} 40 \mathrm{~B}$ & $1.433(19)$ \\
\hline $\mathrm{C} 20-\mathrm{C} 21$ & $1.386(5)$ & $\mathrm{C} 40 \mathrm{~B}-\mathrm{S} 2 \mathrm{~B}$ & $1.678(13)$ \\
\hline $\mathrm{C} 21-\mathrm{C} 22$ & $1.505(4)$ & $\mathrm{C} 40 \mathrm{~B}-\mathrm{H} 40 \mathrm{~B}$ & 0.9500 \\
\hline $\mathrm{C} 22-\mathrm{O} 3$ & $1.274(4)$ & $\mathrm{C} 41 \mathrm{~B}-\mathrm{C} 42 \mathrm{~B}$ & $1.46(2)$ \\
\hline $\mathrm{C} 22-\mathrm{C} 23$ & $1.389(5)$ & $\mathrm{C} 41 \mathrm{~B}-\mathrm{O} 7 \mathrm{~B}$ & $1.47(2)$ \\
\hline $\mathrm{C} 23-\mathrm{C} 24$ & $1.396(5)$ & $\mathrm{C} 41 \mathrm{~B}-\mathrm{H} 41 \mathrm{C}$ & 0.9900 \\
\hline $\mathrm{C} 23-\mathrm{H} 23$ & 0.9500 & $\mathrm{C} 41 \mathrm{~B}-\mathrm{H} 41 \mathrm{D}$ & 0.9900 \\
\hline $\mathrm{C} 24-\mathrm{O} 4$ & $1.268(4)$ & $\mathrm{C} 42 \mathrm{~B}-\mathrm{O} 8 \mathrm{~B}$ & $1.48(2)$ \\
\hline $\mathrm{C} 24-\mathrm{C} 25$ & $1.501(4)$ & $\mathrm{C} 42 \mathrm{~B}-\mathrm{H} 42 \mathrm{C}$ & 0.9900 \\
\hline $\mathrm{C} 25-\mathrm{C} 26$ & $1.394(5)$ & $\mathrm{C} 42 \mathrm{~B}-\mathrm{H} 42 \mathrm{D}$ & 0.9900 \\
\hline $\mathrm{C} 25-\mathrm{C} 30$ & $1.398(5)$ & $\mathrm{C} 43-\mathrm{C} 44$ & $1.365(6)$ \\
\hline $\mathrm{C} 26-\mathrm{F} 16$ & $1.341(4)$ & $\mathrm{C} 43-\mathrm{S} 3$ & $1.745(5)$ \\
\hline $\mathrm{C} 26-\mathrm{C} 27$ & $1.382(5)$ & $\mathrm{C} 43-\mathrm{H} 43$ & 0.9500 \\
\hline $\mathrm{C} 27-\mathrm{F} 17$ & $1.339(4)$ & $\mathrm{C} 44-\mathrm{O} 9$ & $1.365(5)$ \\
\hline $\mathrm{C} 27-\mathrm{C} 28$ & $1.381(5)$ & $\mathrm{C} 44-\mathrm{C} 45$ & $1.399(6)$ \\
\hline $\mathrm{C} 28-\mathrm{F} 18$ & $1.333(4)$ & $\mathrm{C} 45-\mathrm{C} 46$ & $1.361(6)$ \\
\hline $\mathrm{C} 28-\mathrm{C} 29$ & $1.375(5)$ & $\mathrm{C} 45-\mathrm{O} 10$ & $1.379(5)$ \\
\hline C29-F19 & $1.338(4)$ & $\mathrm{C} 46-\mathrm{S} 3$ & $1.652(6)$ \\
\hline $\mathrm{C} 29-\mathrm{C} 30$ & $1.384(5)$ & $\mathrm{C} 46-\mathrm{H} 46$ & 0.9500 \\
\hline $\mathrm{C} 30-\mathrm{F} 20$ & $1.339(4)$ & $\mathrm{C} 47-\mathrm{O} 9$ & $1.413(5)$ \\
\hline $\mathrm{Cu} 1-\mathrm{O} 3$ & $1.923(2)$ & $\mathrm{C} 47-\mathrm{C} 48$ & $1.448(6)$ \\
\hline $\mathrm{Cu} 1-\mathrm{O} 4$ & $1.928(2)$ & $\mathrm{C} 47-\mathrm{H} 47 \mathrm{~A}$ & 0.9900 \\
\hline $\mathrm{Cu} 1-\mathrm{O} 1$ & $1.940(2)$ & C47-H47B & 0.9900 \\
\hline $\mathrm{Cu} 1-\mathrm{O} 2$ & $1.941(2)$ & $\mathrm{C} 48-\mathrm{O} 10$ & $1.442(5)$ \\
\hline $\mathrm{Cu} 1-\mathrm{O}^{\mathrm{i}}$ & $2.711(2)$ & $\mathrm{C} 48-\mathrm{H} 48 \mathrm{~A}$ & 0.9900 \\
\hline $\mathrm{Cu} 1-\mathrm{O} 6$ & $2.421(2)$ & C48-H48B & 0.9900 \\
\hline
\end{tabular}




\begin{tabular}{|c|c|c|c|}
\hline $\mathrm{F} 1-\mathrm{C} 1-\mathrm{C} 2$ & $118.4(3)$ & $\mathrm{C} 31-\mathrm{C} 32-\mathrm{O} 5$ & $124.4(3)$ \\
\hline $\mathrm{F} 1-\mathrm{C} 1-\mathrm{C} 6$ & $119.6(3)$ & $\mathrm{C} 31-\mathrm{C} 32-\mathrm{C} 33$ & $113.0(3)$ \\
\hline $\mathrm{C} 2-\mathrm{C} 1-\mathrm{C} 6$ & $122.0(3)$ & $\mathrm{O} 5-\mathrm{C} 32-\mathrm{C} 33$ & $122.6(3)$ \\
\hline $\mathrm{F} 2-\mathrm{C} 2-\mathrm{C} 3$ & $119.7(3)$ & $\mathrm{C} 34-\mathrm{C} 33-\mathrm{O} 6$ & $124.1(3)$ \\
\hline $\mathrm{F} 2-\mathrm{C} 2-\mathrm{C} 1$ & $120.8(3)$ & $\mathrm{C} 34-\mathrm{C} 33-\mathrm{C} 32$ & $113.4(3)$ \\
\hline $\mathrm{C} 3-\mathrm{C} 2-\mathrm{C} 1$ & $119.6(3)$ & $\mathrm{O} 6-\mathrm{C} 33-\mathrm{C} 32$ & $122.5(3)$ \\
\hline $\mathrm{F} 3-\mathrm{C} 3-\mathrm{C} 4$ & $119.9(3)$ & $\mathrm{C} 33-\mathrm{C} 34-\mathrm{S} 1$ & $110.2(3)$ \\
\hline $\mathrm{F} 3-\mathrm{C} 3-\mathrm{C} 2$ & $120.0(3)$ & $\mathrm{C} 33-\mathrm{C} 34-\mathrm{H} 34$ & 124.9 \\
\hline $\mathrm{C} 4-\mathrm{C} 3-\mathrm{C} 2$ & $120.2(3)$ & $\mathrm{S} 1-\mathrm{C} 34-\mathrm{H} 34$ & 124.9 \\
\hline $\mathrm{F} 4-\mathrm{C} 4-\mathrm{C} 3$ & $120.2(3)$ & $\mathrm{O} 5-\mathrm{C} 35-\mathrm{C} 36$ & $111.3(3)$ \\
\hline $\mathrm{F} 4-\mathrm{C} 4-\mathrm{C} 5$ & $120.5(3)$ & $\mathrm{O} 5-\mathrm{C} 35-\mathrm{H} 35 \mathrm{~A}$ & 109.4 \\
\hline $\mathrm{C} 3-\mathrm{C} 4-\mathrm{C} 5$ & $119.3(3)$ & $\mathrm{C} 36-\mathrm{C} 35-\mathrm{H} 35 \mathrm{~A}$ & 109.4 \\
\hline $\mathrm{F} 5-\mathrm{C} 5-\mathrm{C} 4$ & $118.0(3)$ & $\mathrm{O} 5-\mathrm{C} 35-\mathrm{H} 35 \mathrm{~B}$ & 109.4 \\
\hline $\mathrm{F} 5-\mathrm{C} 5-\mathrm{C} 6$ & $119.6(3)$ & $\mathrm{C} 36-\mathrm{C} 35-\mathrm{H} 35 \mathrm{~B}$ & 109.4 \\
\hline $\mathrm{C} 4-\mathrm{C} 5-\mathrm{C} 6$ & $122.4(3)$ & $\mathrm{H} 35 \mathrm{~A}-\mathrm{C} 35-\mathrm{H} 35 \mathrm{~B}$ & 108.0 \\
\hline $\mathrm{C} 1-\mathrm{C} 6-\mathrm{C} 5$ & $116.6(3)$ & $\mathrm{O} 6-\mathrm{C} 36-\mathrm{C} 35$ & $110.6(3)$ \\
\hline $\mathrm{C} 1-\mathrm{C} 6-\mathrm{C} 7$ & $121.8(3)$ & $\mathrm{O} 6-\mathrm{C} 36-\mathrm{H} 36 \mathrm{~A}$ & 109.5 \\
\hline $\mathrm{C} 5-\mathrm{C} 6-\mathrm{C} 7$ & $121.6(3)$ & $\mathrm{C} 35-\mathrm{C} 36-\mathrm{H} 36 \mathrm{~A}$ & 109.5 \\
\hline $\mathrm{O} 1-\mathrm{C} 7-\mathrm{C} 8$ & $127.3(3)$ & $\mathrm{O} 6-\mathrm{C} 36-\mathrm{H} 36 \mathrm{~B}$ & 109.5 \\
\hline $\mathrm{O} 1-\mathrm{C} 7-\mathrm{C} 6$ & $115.8(3)$ & $\mathrm{C} 35-\mathrm{C} 36-\mathrm{H} 36 \mathrm{~B}$ & 109.5 \\
\hline $\mathrm{C} 8-\mathrm{C} 7-\mathrm{C} 6$ & $117.0(3)$ & $\mathrm{H} 36 \mathrm{~A}-\mathrm{C} 36-\mathrm{H} 36 \mathrm{~B}$ & 108.1 \\
\hline $\mathrm{C} 7-\mathrm{C} 8-\mathrm{C} 9$ & $123.5(3)$ & $\mathrm{C} 32-\mathrm{O} 5-\mathrm{C} 35$ & $111.7(2)$ \\
\hline $\mathrm{C} 7-\mathrm{C} 8-\mathrm{H} 8$ & 118.2 & $\mathrm{C} 33-\mathrm{O} 6-\mathrm{C} 36$ & $111.5(2)$ \\
\hline $\mathrm{C} 9-\mathrm{C} 8-\mathrm{H} 8$ & 118.2 & $\mathrm{C} 33-\mathrm{O} 6-\mathrm{Cu} 1$ & $121.57(18)$ \\
\hline $\mathrm{O} 2-\mathrm{C} 9-\mathrm{C} 8$ & $125.7(3)$ & $\mathrm{C} 36-\mathrm{O} 6-\mathrm{Cu} 1$ & $121.90(18)$ \\
\hline $\mathrm{O} 2-\mathrm{C} 9-\mathrm{C} 10$ & $115.2(3)$ & $\mathrm{C} 31-\mathrm{S} 1-\mathrm{C} 34$ & $92.92(16)$ \\
\hline $\mathrm{C} 8-\mathrm{C} 9-\mathrm{C} 10$ & $119.1(3)$ & $\mathrm{C} 38 \mathrm{~A}-\mathrm{C} 37 \mathrm{~A}-\mathrm{S} 2 \mathrm{~A}$ & $115.0(8)$ \\
\hline $\mathrm{C} 15-\mathrm{C} 10-\mathrm{C} 11$ & $115.5(3)$ & $\mathrm{C} 38 \mathrm{~A}-\mathrm{C} 37 \mathrm{~A}-\mathrm{H} 37 \mathrm{~A}$ & 122.5 \\
\hline $\mathrm{C} 15-\mathrm{C} 10-\mathrm{C} 9$ & $123.3(3)$ & $\mathrm{S} 2 \mathrm{~A}-\mathrm{C} 37 \mathrm{~A}-\mathrm{H} 37 \mathrm{~A}$ & 122.5 \\
\hline $\mathrm{C} 11-\mathrm{C} 10-\mathrm{C} 9$ & $121.2(3)$ & O7A $-\mathrm{C} 38 \mathrm{~A}-\mathrm{C} 39 \mathrm{~A}$ & $123.2(9)$ \\
\hline $\mathrm{F} 6-\mathrm{C} 11-\mathrm{C} 12$ & $116.9(3)$ & O7A $-\mathrm{C} 38 \mathrm{~A}-\mathrm{C} 37 \mathrm{~A}$ & $129.5(10)$ \\
\hline $\mathrm{F} 6-\mathrm{C} 11-\mathrm{C} 10$ & $120.3(3)$ & $\mathrm{C} 39 \mathrm{~A}-\mathrm{C} 38 \mathrm{~A}-\mathrm{C} 37 \mathrm{~A}$ & $107.3(11)$ \\
\hline $\mathrm{C} 12-\mathrm{C} 11-\mathrm{C} 10$ & $122.7(3)$ & $\mathrm{C} 40 \mathrm{~A}-\mathrm{C} 39 \mathrm{~A}-\mathrm{O} 8 \mathrm{~A}$ & $126.2(9)$ \\
\hline $\mathrm{F} 7-\mathrm{C} 12-\mathrm{C} 13$ & $120.3(3)$ & $\mathrm{C} 40 \mathrm{~A}-\mathrm{C} 39 \mathrm{~A}-\mathrm{C} 38 \mathrm{~A}$ & $112.4(9)$ \\
\hline $\mathrm{F} 7-\mathrm{C} 12-\mathrm{C} 11$ & $120.4(3)$ & O8A $-\mathrm{C} 39 \mathrm{~A}-\mathrm{C} 38 \mathrm{~A}$ & $121.4(10)$ \\
\hline $\mathrm{C} 13-\mathrm{C} 12-\mathrm{C} 11$ & $119.3(3)$ & $\mathrm{C} 39 \mathrm{~A}-\mathrm{C} 40 \mathrm{~A}-\mathrm{S} 2 \mathrm{~A}$ & $113.7(9)$ \\
\hline $\mathrm{F} 8-\mathrm{C} 13-\mathrm{C} 12$ & $120.4(3)$ & $\mathrm{C} 39 \mathrm{~A}-\mathrm{C} 40 \mathrm{~A}-\mathrm{H} 40 \mathrm{~A}$ & 123.2 \\
\hline $\mathrm{F} 8-\mathrm{C} 13-\mathrm{C} 14$ & $119.6(3)$ & $\mathrm{S} 2 \mathrm{~A}-\mathrm{C} 40 \mathrm{~A}-\mathrm{H} 40 \mathrm{~A}$ & 123.2 \\
\hline $\mathrm{C} 12-\mathrm{C} 13-\mathrm{C} 14$ & $120.0(3)$ & $\mathrm{O} 7 \mathrm{~A}-\mathrm{C} 41 \mathrm{~A}-\mathrm{C} 42 \mathrm{~A}$ & $110.5(8)$ \\
\hline $\mathrm{F} 9-\mathrm{C} 14-\mathrm{C} 13$ & $120.6(3)$ & $\mathrm{O} 7 \mathrm{~A}-\mathrm{C} 41 \mathrm{~A}-\mathrm{H} 41 \mathrm{~A}$ & 109.5 \\
\hline $\mathrm{F} 9-\mathrm{C} 14-\mathrm{C} 15$ & $119.7(3)$ & $\mathrm{C} 42 \mathrm{~A}-\mathrm{C} 41 \mathrm{~A}-\mathrm{H} 41 \mathrm{~A}$ & 109.5 \\
\hline $\mathrm{C} 13-\mathrm{C} 14-\mathrm{C} 15$ & $119.7(3)$ & $\mathrm{O} 7 \mathrm{~A}-\mathrm{C} 41 \mathrm{~A}-\mathrm{H} 41 \mathrm{~B}$ & 109.5 \\
\hline $\mathrm{F} 10-\mathrm{C} 15-\mathrm{C} 14$ & $116.6(3)$ & $\mathrm{C} 42 \mathrm{~A}-\mathrm{C} 41 \mathrm{~A}-\mathrm{H} 41 \mathrm{~B}$ & 109.5 \\
\hline $\mathrm{F} 10-\mathrm{C} 15-\mathrm{C} 10$ & $120.7(3)$ & $\mathrm{H} 41 \mathrm{~A}-\mathrm{C} 41 \mathrm{~A}-\mathrm{H} 41 \mathrm{~B}$ & 108.1 \\
\hline $\mathrm{C} 14-\mathrm{C} 15-\mathrm{C} 10$ & $122.7(3)$ & $\mathrm{O} 8 \mathrm{~A}-\mathrm{C} 42 \mathrm{~A}-\mathrm{C} 41 \mathrm{~A}$ & $111.8(7)$ \\
\hline $\mathrm{F} 11-\mathrm{C} 16-\mathrm{C} 17$ & $117.7(3)$ & $\mathrm{O} 8 \mathrm{~A}-\mathrm{C} 42 \mathrm{~A}-\mathrm{H} 42 \mathrm{~A}$ & 109.3 \\
\hline $\mathrm{F} 11-\mathrm{C} 16-\mathrm{C} 21$ & $119.8(3)$ & $\mathrm{C} 41 \mathrm{~A}-\mathrm{C} 42 \mathrm{~A}-\mathrm{H} 42 \mathrm{~A}$ & 109.3 \\
\hline
\end{tabular}




\begin{tabular}{|c|c|}
\hline $\mathrm{C} 17-\mathrm{C} 16-\mathrm{C} 21$ & $122.5(3)$ \\
\hline $\mathrm{F} 12-\mathrm{C} 17-\mathrm{C} 16$ & $120.5(3)$ \\
\hline $\mathrm{F} 12-\mathrm{C} 17-\mathrm{C} 18$ & $120.0(3)$ \\
\hline $\mathrm{C} 16-\mathrm{C} 17-\mathrm{C} 18$ & $119.5(3)$ \\
\hline $\mathrm{F} 13-\mathrm{C} 18-\mathrm{C} 17$ & $120.0(3)$ \\
\hline $\mathrm{F} 13-\mathrm{C} 18-\mathrm{C} 19$ & $120.4(3)$ \\
\hline $\mathrm{C} 17-\mathrm{C} 18-\mathrm{C} 19$ & $119.6(3)$ \\
\hline $\mathrm{F} 14-\mathrm{C} 19-\mathrm{C} 20$ & $119.7(3)$ \\
\hline $\mathrm{F} 14-\mathrm{C} 19-\mathrm{C} 18$ & $120.4(3)$ \\
\hline $\mathrm{C} 20-\mathrm{C} 19-\mathrm{C} 18$ & $119.9(3)$ \\
\hline $\mathrm{F} 15-\mathrm{C} 20-\mathrm{C} 19$ & $117.5(3)$ \\
\hline $\mathrm{F} 15-\mathrm{C} 20-\mathrm{C} 21$ & $120.5(3)$ \\
\hline $\mathrm{C} 19-\mathrm{C} 20-\mathrm{C} 21$ & $122.0(3)$ \\
\hline $\mathrm{C} 20-\mathrm{C} 21-\mathrm{C} 16$ & $116.6(3)$ \\
\hline $\mathrm{C} 20-\mathrm{C} 21-\mathrm{C} 22$ & $121.7(3)$ \\
\hline $\mathrm{C} 16-\mathrm{C} 21-\mathrm{C} 22$ & $121.6(3)$ \\
\hline $\mathrm{O} 3-\mathrm{C} 22-\mathrm{C} 23$ & $126.8(3)$ \\
\hline $\mathrm{O} 3-\mathrm{C} 22-\mathrm{C} 21$ & $114.3(3)$ \\
\hline $\mathrm{C} 23-\mathrm{C} 22-\mathrm{C} 21$ & $119.0(3)$ \\
\hline $\mathrm{C} 22-\mathrm{C} 23-\mathrm{C} 24$ & $122.9(3)$ \\
\hline $\mathrm{C} 22-\mathrm{C} 23-\mathrm{H} 23$ & 118.5 \\
\hline $\mathrm{C} 24-\mathrm{C} 23-\mathrm{H} 23$ & 118.5 \\
\hline $\mathrm{O} 4-\mathrm{C} 24-\mathrm{C} 23$ & $126.0(3)$ \\
\hline $\mathrm{O} 4-\mathrm{C} 24-\mathrm{C} 25$ & $115.4(3)$ \\
\hline $\mathrm{C} 23-\mathrm{C} 24-\mathrm{C} 25$ & $118.7(3)$ \\
\hline $\mathrm{C} 26-\mathrm{C} 25-\mathrm{C} 30$ & $116.3(3)$ \\
\hline $\mathrm{C} 26-\mathrm{C} 25-\mathrm{C} 24$ & $121.8(3)$ \\
\hline $\mathrm{C} 30-\mathrm{C} 25-\mathrm{C} 24$ & $121.7(3)$ \\
\hline $\mathrm{F} 16-\mathrm{C} 26-\mathrm{C} 27$ & $117.8(3)$ \\
\hline $\mathrm{F} 16-\mathrm{C} 26-\mathrm{C} 25$ & $120.2(3)$ \\
\hline $\mathrm{C} 27-\mathrm{C} 26-\mathrm{C} 25$ & $122.0(3)$ \\
\hline $\mathrm{F} 17-\mathrm{C} 27-\mathrm{C} 28$ & $119.7(3)$ \\
\hline $\mathrm{F} 17-\mathrm{C} 27-\mathrm{C} 26$ & $120.3(3)$ \\
\hline $\mathrm{C} 28-\mathrm{C} 27-\mathrm{C} 26$ & $120.0(3)$ \\
\hline $\mathrm{F} 18-\mathrm{C} 28-\mathrm{C} 29$ & $120.2(3)$ \\
\hline $\mathrm{F} 18-\mathrm{C} 28-\mathrm{C} 27$ & $120.0(3)$ \\
\hline $\mathrm{C} 29-\mathrm{C} 28-\mathrm{C} 27$ & $119.7(3)$ \\
\hline $\mathrm{F} 19-\mathrm{C} 29-\mathrm{C} 28$ & $120.2(3)$ \\
\hline $\mathrm{F} 19-\mathrm{C} 29-\mathrm{C} 30$ & $120.0(3)$ \\
\hline $\mathrm{C} 28-\mathrm{C} 29-\mathrm{C} 30$ & $119.7(3)$ \\
\hline $\mathrm{F} 20-\mathrm{C} 30-\mathrm{C} 29$ & $117.2(3)$ \\
\hline $\mathrm{F} 20-\mathrm{C} 30-\mathrm{C} 25$ & $120.6(3)$ \\
\hline $\mathrm{C} 29-\mathrm{C} 30-\mathrm{C} 25$ & $122.2(3)$ \\
\hline $\mathrm{O} 3-\mathrm{Cu} 1-\mathrm{O} 4$ & $93.47(9)$ \\
\hline $\mathrm{O} 3-\mathrm{Cu} 1-\mathrm{O} 1$ & $178.39(9)$ \\
\hline $\mathrm{O} 4-\mathrm{Cu} 1-\mathrm{O} 1$ & $87.48(9)$ \\
\hline $\mathrm{O} 3-\mathrm{Cu} 1-\mathrm{O} 2$ & $85.82(9)$ \\
\hline $\mathrm{O} 4-\mathrm{Cu} 1-\mathrm{O} 2$ & $175.70(9)$ \\
\hline
\end{tabular}

\begin{tabular}{|c|c|}
\hline $\mathrm{O} 8 \mathrm{~A}-\mathrm{C} 42 \mathrm{~A}-\mathrm{H} 42 \mathrm{~B}$ & 109.3 \\
\hline $\mathrm{C} 41 \mathrm{~A}-\mathrm{C} 42 \mathrm{~A}-\mathrm{H} 42 \mathrm{~B}$ & 109.3 \\
\hline $\mathrm{H} 42 \mathrm{~A}-\mathrm{C} 42 \mathrm{~A}-\mathrm{H} 42 \mathrm{~B}$ & 107.9 \\
\hline $\mathrm{C} 38 \mathrm{~A}-\mathrm{O} 7 \mathrm{~A}-\mathrm{C} 41 \mathrm{~A}$ & $111.8(6)$ \\
\hline $\mathrm{C} 39 \mathrm{~A}-\mathrm{O} 8 \mathrm{~A}-\mathrm{C} 42 \mathrm{~A}$ & $113.2(7)$ \\
\hline $\mathrm{C} 37 \mathrm{~A}-\mathrm{S} 2 \mathrm{~A}-\mathrm{C} 40 \mathrm{~A}$ & $91.5(6)$ \\
\hline $\mathrm{C} 38 \mathrm{~B}-\mathrm{C} 37 \mathrm{~B}-\mathrm{S} 2 \mathrm{~B}$ & $107.0(11)$ \\
\hline $\mathrm{C} 38 \mathrm{~B}-\mathrm{C} 37 \mathrm{~B}-\mathrm{H} 37 \mathrm{~B}$ & 126.5 \\
\hline $\mathrm{S} 2 \mathrm{~B}-\mathrm{C} 37 \mathrm{~B}-\mathrm{H} 37 \mathrm{~B}$ & 126.5 \\
\hline $\mathrm{C} 37 \mathrm{~B}-\mathrm{C} 38 \mathrm{~B}-\mathrm{O} 7 \mathrm{~B}$ & $120.8(17)$ \\
\hline $\mathrm{C} 37 \mathrm{~B}-\mathrm{C} 38 \mathrm{~B}-\mathrm{C} 39 \mathrm{~B}$ & $118.6(14)$ \\
\hline $\mathrm{O} 7 \mathrm{~B}-\mathrm{C} 38 \mathrm{~B}-\mathrm{C} 39 \mathrm{~B}$ & $120.6(15)$ \\
\hline $\mathrm{O} 8 \mathrm{~B}-\mathrm{C} 39 \mathrm{~B}-\mathrm{C} 40 \mathrm{~B}$ & $121.6(13)$ \\
\hline $\mathrm{O} 8 \mathrm{~B}-\mathrm{C} 39 \mathrm{~B}-\mathrm{C} 38 \mathrm{~B}$ & $126.6(14)$ \\
\hline $\mathrm{C} 40 \mathrm{~B}-\mathrm{C} 39 \mathrm{~B}-\mathrm{C} 38 \mathrm{~B}$ & $111.8(12)$ \\
\hline $\mathrm{C} 39 \mathrm{~B}-\mathrm{C} 40 \mathrm{~B}-\mathrm{S} 2 \mathrm{~B}$ & $108.2(10)$ \\
\hline $\mathrm{C} 39 \mathrm{~B}-\mathrm{C} 40 \mathrm{~B}-\mathrm{H} 40 \mathrm{~B}$ & 125.9 \\
\hline $\mathrm{S} 2 \mathrm{~B}-\mathrm{C} 40 \mathrm{~B}-\mathrm{H} 40 \mathrm{~B}$ & 125.9 \\
\hline $\mathrm{C} 42 \mathrm{~B}-\mathrm{C} 41 \mathrm{~B}-\mathrm{O} 7 \mathrm{~B}$ & $113.7(12)$ \\
\hline $\mathrm{C} 42 \mathrm{~B}-\mathrm{C} 41 \mathrm{~B}-\mathrm{H} 41 \mathrm{C}$ & 108.8 \\
\hline $\mathrm{O} 7 \mathrm{~B}-\mathrm{C} 41 \mathrm{~B}-\mathrm{H} 41 \mathrm{C}$ & 108.8 \\
\hline $\mathrm{C} 42 \mathrm{~B}-\mathrm{C} 41 \mathrm{~B}-\mathrm{H} 41 \mathrm{D}$ & 108.8 \\
\hline $\mathrm{O} 7 \mathrm{~B}-\mathrm{C} 41 \mathrm{~B}-\mathrm{H} 41 \mathrm{D}$ & 108.8 \\
\hline $\mathrm{H} 41 \mathrm{C}-\mathrm{C} 41 \mathrm{~B}-\mathrm{H} 41 \mathrm{D}$ & 107.7 \\
\hline $\mathrm{C} 41 \mathrm{~B}-\mathrm{C} 42 \mathrm{~B}-\mathrm{O} 8 \mathrm{~B}$ & $107.9(13)$ \\
\hline $\mathrm{C} 41 \mathrm{~B}-\mathrm{C} 42 \mathrm{~B}-\mathrm{H} 42 \mathrm{C}$ & 110.1 \\
\hline $\mathrm{O} 8 \mathrm{~B}-\mathrm{C} 42 \mathrm{~B}-\mathrm{H} 42 \mathrm{C}$ & 110.1 \\
\hline $\mathrm{C} 41 \mathrm{~B}-\mathrm{C} 42 \mathrm{~B}-\mathrm{H} 42 \mathrm{D}$ & 110.1 \\
\hline $\mathrm{O} 8 \mathrm{~B}-\mathrm{C} 42 \mathrm{~B}-\mathrm{H} 42 \mathrm{D}$ & 110.1 \\
\hline $\mathrm{H} 42 \mathrm{C}-\mathrm{C} 42 \mathrm{~B}-\mathrm{H} 42 \mathrm{D}$ & 108.4 \\
\hline $\mathrm{C} 38 \mathrm{~B}-\mathrm{O} 7 \mathrm{~B}-\mathrm{C} 41 \mathrm{~B}$ & $111.0(13)$ \\
\hline $\mathrm{C} 39 \mathrm{~B}-\mathrm{O} 8 \mathrm{~B}-\mathrm{C} 42 \mathrm{~B}$ & $106.6(13)$ \\
\hline $\mathrm{C} 40 \mathrm{~B}-\mathrm{S} 2 \mathrm{~B}-\mathrm{C} 37 \mathrm{~B}$ & $94.3(6)$ \\
\hline $\mathrm{C} 44-\mathrm{C} 43-\mathrm{S} 3$ & $109.2(4)$ \\
\hline $\mathrm{C} 44-\mathrm{C} 43-\mathrm{H} 43$ & 125.4 \\
\hline $\mathrm{S} 3-\mathrm{C} 43-\mathrm{H} 43$ & 125.4 \\
\hline $\mathrm{O} 9-\mathrm{C} 44-\mathrm{C} 43$ & $124.7(4)$ \\
\hline $\mathrm{O} 9-\mathrm{C} 44-\mathrm{C} 45$ & $122.4(4)$ \\
\hline $\mathrm{C} 43-\mathrm{C} 44-\mathrm{C} 45$ & $112.8(4)$ \\
\hline $\mathrm{C} 46-\mathrm{C} 45-\mathrm{O} 10$ & $124.1(4)$ \\
\hline $\mathrm{C} 46-\mathrm{C} 45-\mathrm{C} 44$ & $112.8(4)$ \\
\hline $\mathrm{O} 10-\mathrm{C} 45-\mathrm{C} 44$ & $123.1(3)$ \\
\hline $\mathrm{C} 45-\mathrm{C} 46-\mathrm{S} 3$ & $112.5(4)$ \\
\hline $\mathrm{C} 45-\mathrm{C} 46-\mathrm{H} 46$ & 123.8 \\
\hline $\mathrm{S} 3-\mathrm{C} 46-\mathrm{H} 46$ & 123.8 \\
\hline $\mathrm{O} 9-\mathrm{C} 47-\mathrm{C} 48$ & $115.2(3)$ \\
\hline $\mathrm{O} 9-\mathrm{C} 47-\mathrm{H} 47 \mathrm{~A}$ & 108.5 \\
\hline $\mathrm{C} 48-\mathrm{C} 47-\mathrm{H} 47 \mathrm{~A}$ & 108.5 \\
\hline
\end{tabular}




\begin{tabular}{|c|c|c|c|}
\hline $\mathrm{O} 1-\mathrm{Cu} 1-\mathrm{O} 2$ & $93.32(9)$ & $\mathrm{O} 9-\mathrm{C} 47-\mathrm{H} 47 \mathrm{~B}$ & 108.5 \\
\hline $\mathrm{O} 3-\mathrm{Cu} 1-\mathrm{O} 6$ & $88.12(9)$ & $\mathrm{C} 48-\mathrm{C} 47-\mathrm{H} 47 \mathrm{~B}$ & 108.5 \\
\hline $\mathrm{O} 4-\mathrm{Cu} 1-\mathrm{O} 6$ & $93.75(8)$ & $\mathrm{H} 47 \mathrm{~A}-\mathrm{C} 47-\mathrm{H} 47 \mathrm{~B}$ & 107.5 \\
\hline $\mathrm{O} 1-\mathrm{Cu} 1-\mathrm{O} 6$ & $90.53(8)$ & $\mathrm{O} 10-\mathrm{C} 48-\mathrm{C} 47$ & $110.4(3)$ \\
\hline $\mathrm{O} 2-\mathrm{Cu} 1-\mathrm{O} 6$ & $90.46(9)$ & $\mathrm{O} 10-\mathrm{C} 48-\mathrm{H} 48 \mathrm{~A}$ & 109.6 \\
\hline $\mathrm{C} 7-\mathrm{O} 1-\mathrm{Cu} 1$ & $123.3(2)$ & $\mathrm{C} 47-\mathrm{C} 48-\mathrm{H} 48 \mathrm{~A}$ & 109.6 \\
\hline $\mathrm{C} 9-\mathrm{O} 2-\mathrm{Cu} 1$ & $125.0(2)$ & $\mathrm{O} 10-\mathrm{C} 48-\mathrm{H} 48 \mathrm{~B}$ & 109.6 \\
\hline $\mathrm{C} 22-\mathrm{O} 3-\mathrm{Cu} 1$ & $124.4(2)$ & $\mathrm{C} 47-\mathrm{C} 48-\mathrm{H} 48 \mathrm{~B}$ & 109.6 \\
\hline $\mathrm{C} 24-\mathrm{O} 4-\mathrm{Cu} 1$ & $123.7(2)$ & $\mathrm{H} 48 \mathrm{~A}-\mathrm{C} 48-\mathrm{H} 48 \mathrm{~B}$ & 108.1 \\
\hline $\mathrm{C} 32-\mathrm{C} 31-\mathrm{S} 1$ & $110.4(2)$ & $\mathrm{C} 44-\mathrm{O} 9-\mathrm{C} 47$ & $111.3(3)$ \\
\hline $\mathrm{C} 32-\mathrm{C} 31-\mathrm{H} 31$ & 124.8 & $\mathrm{C} 45-\mathrm{O} 10-\mathrm{C} 48$ & $111.3(3)$ \\
\hline $\mathrm{S} 1-\mathrm{C} 31-\mathrm{H} 31$ & 124.8 & $\mathrm{C} 46-\mathrm{S} 3-\mathrm{C} 43$ & $92.6(2)$ \\
\hline $\mathrm{F} 1-\mathrm{C} 1-\mathrm{C} 2-\mathrm{F} 2$ & $0.8(5)$ & $\mathrm{F} 16-\mathrm{C} 26-\mathrm{C} 27-\mathrm{F} 17$ & $1.1(5)$ \\
\hline $\mathrm{C} 6-\mathrm{C} 1-\mathrm{C} 2-\mathrm{F} 2$ & $178.8(3)$ & $\mathrm{C} 25-\mathrm{C} 26-\mathrm{C} 27-\mathrm{F} 17$ & $179.2(3)$ \\
\hline $\mathrm{F} 1-\mathrm{C} 1-\mathrm{C} 2-\mathrm{C} 3$ & $-178.7(3)$ & $\mathrm{F} 16-\mathrm{C} 26-\mathrm{C} 27-\mathrm{C} 28$ & $-179.3(3)$ \\
\hline $\mathrm{C} 6-\mathrm{C} 1-\mathrm{C} 2-\mathrm{C} 3$ & $-0.7(5)$ & $\mathrm{C} 25-\mathrm{C} 26-\mathrm{C} 27-\mathrm{C} 28$ & $-1.3(5)$ \\
\hline $\mathrm{F} 2-\mathrm{C} 2-\mathrm{C} 3-\mathrm{F} 3$ & $0.4(5)$ & $\mathrm{F} 17-\mathrm{C} 27-\mathrm{C} 28-\mathrm{F} 18$ & $0.2(5)$ \\
\hline $\mathrm{C} 1-\mathrm{C} 2-\mathrm{C} 3-\mathrm{F} 3$ & $180.0(3)$ & $\mathrm{C} 26-\mathrm{C} 27-\mathrm{C} 28-\mathrm{F} 18$ & $-179.3(3)$ \\
\hline $\mathrm{F} 2-\mathrm{C} 2-\mathrm{C} 3-\mathrm{C} 4$ & $-179.6(3)$ & $\mathrm{F} 17-\mathrm{C} 27-\mathrm{C} 28-\mathrm{C} 29$ & $-179.4(3)$ \\
\hline $\mathrm{C} 1-\mathrm{C} 2-\mathrm{C} 3-\mathrm{C} 4$ & $-0.1(5)$ & $\mathrm{C} 26-\mathrm{C} 27-\mathrm{C} 28-\mathrm{C} 29$ & $1.0(5)$ \\
\hline $\mathrm{F} 3-\mathrm{C} 3-\mathrm{C} 4-\mathrm{F} 4$ & $0.2(5)$ & $\mathrm{F} 18-\mathrm{C} 28-\mathrm{C} 29-\mathrm{F} 19$ & $1.9(5)$ \\
\hline $\mathrm{C} 2-\mathrm{C} 3-\mathrm{C} 4-\mathrm{F} 4$ & $-179.7(3)$ & $\mathrm{C} 27-\mathrm{C} 28-\mathrm{C} 29-\mathrm{F} 19$ & $-178.5(3)$ \\
\hline $\mathrm{F} 3-\mathrm{C} 3-\mathrm{C} 4-\mathrm{C} 5$ & $-178.9(3)$ & $\mathrm{F} 18-\mathrm{C} 28-\mathrm{C} 29-\mathrm{C} 30$ & $-179.6(3)$ \\
\hline $\mathrm{C} 2-\mathrm{C} 3-\mathrm{C} 4-\mathrm{C} 5$ & $1.1(5)$ & $\mathrm{C} 27-\mathrm{C} 28-\mathrm{C} 29-\mathrm{C} 30$ & $0.0(5)$ \\
\hline $\mathrm{F} 4-\mathrm{C} 4-\mathrm{C} 5-\mathrm{F} 5$ & $-1.7(5)$ & $\mathrm{F} 19-\mathrm{C} 29-\mathrm{C} 30-\mathrm{F} 20$ & $-0.5(5)$ \\
\hline $\mathrm{C} 3-\mathrm{C} 4-\mathrm{C} 5-\mathrm{F} 5$ & $177.4(3)$ & $\mathrm{C} 28-\mathrm{C} 29-\mathrm{C} 30-\mathrm{F} 20$ & $-179.0(3)$ \\
\hline $\mathrm{F} 4-\mathrm{C} 4-\mathrm{C} 5-\mathrm{C} 6$ & $179.5(3)$ & $\mathrm{F} 19-\mathrm{C} 29-\mathrm{C} 30-\mathrm{C} 25$ & $177.6(3)$ \\
\hline $\mathrm{C} 3-\mathrm{C} 4-\mathrm{C} 5-\mathrm{C} 6$ & $-1.4(5)$ & $\mathrm{C} 28-\mathrm{C} 29-\mathrm{C} 30-\mathrm{C} 25$ & $-0.9(5)$ \\
\hline $\mathrm{F} 1-\mathrm{C} 1-\mathrm{C} 6-\mathrm{C} 5$ & $178.5(3)$ & $\mathrm{C} 26-\mathrm{C} 25-\mathrm{C} 30-\mathrm{F} 20$ & $178.8(3)$ \\
\hline $\mathrm{C} 2-\mathrm{C} 1-\mathrm{C} 6-\mathrm{C} 5$ & $0.5(5)$ & $\mathrm{C} 24-\mathrm{C} 25-\mathrm{C} 30-\mathrm{F} 20$ & $4.0(5)$ \\
\hline $\mathrm{F} 1-\mathrm{C} 1-\mathrm{C} 6-\mathrm{C} 7$ & $-0.2(5)$ & $\mathrm{C} 26-\mathrm{C} 25-\mathrm{C} 30-\mathrm{C} 29$ & $0.7(5)$ \\
\hline $\mathrm{C} 2-\mathrm{C} 1-\mathrm{C} 6-\mathrm{C} 7$ & $-178.2(3)$ & $\mathrm{C} 24-\mathrm{C} 25-\mathrm{C} 30-\mathrm{C} 29$ & $-174.1(3)$ \\
\hline $\mathrm{F} 5-\mathrm{C} 5-\mathrm{C} 6-\mathrm{C} 1$ & $-178.2(3)$ & $\mathrm{C} 8-\mathrm{C} 7-\mathrm{O} 1-\mathrm{Cu} 1$ & $12.6(4)$ \\
\hline $\mathrm{C} 4-\mathrm{C} 5-\mathrm{C} 6-\mathrm{C} 1$ & $0.6(5)$ & $\mathrm{C} 6-\mathrm{C} 7-\mathrm{O} 1-\mathrm{Cu} 1$ & $-168.61(19)$ \\
\hline $\mathrm{F} 5-\mathrm{C} 5-\mathrm{C} 6-\mathrm{C} 7$ & $0.5(4)$ & $\mathrm{C} 8-\mathrm{C} 9-\mathrm{O} 2-\mathrm{Cu} 1$ & $-4.8(4)$ \\
\hline $\mathrm{C} 4-\mathrm{C} 5-\mathrm{C} 6-\mathrm{C} 7$ & $179.3(3)$ & $\mathrm{C} 10-\mathrm{C} 9-\mathrm{O} 2-\mathrm{Cu} 1$ & $173.90(19)$ \\
\hline $\mathrm{C} 1-\mathrm{C} 6-\mathrm{C} 7-\mathrm{O} 1$ & $-61.9(4)$ & $\mathrm{C} 23-\mathrm{C} 22-\mathrm{O} 3-\mathrm{Cu} 1$ & $-1.0(5)$ \\
\hline $\mathrm{C} 5-\mathrm{C} 6-\mathrm{C} 7-\mathrm{O} 1$ & $119.4(3)$ & $\mathrm{C} 21-\mathrm{C} 22-\mathrm{O} 3-\mathrm{Cu} 1$ & $177.7(2)$ \\
\hline $\mathrm{C} 1-\mathrm{C} 6-\mathrm{C} 7-\mathrm{C} 8$ & $117.0(3)$ & $\mathrm{C} 23-\mathrm{C} 24-\mathrm{O} 4-\mathrm{Cu} 1$ & $19.4(4)$ \\
\hline $\mathrm{C} 5-\mathrm{C} 6-\mathrm{C} 7-\mathrm{C} 8$ & $-61.6(4)$ & $\mathrm{C} 25-\mathrm{C} 24-\mathrm{O} 4-\mathrm{Cu} 1$ & $-159.1(2)$ \\
\hline $\mathrm{O} 1-\mathrm{C} 7-\mathrm{C} 8-\mathrm{C} 9$ & $-1.3(5)$ & $\mathrm{S} 1-\mathrm{C} 31-\mathrm{C} 32-\mathrm{O} 5$ & $-178.2(2)$ \\
\hline $\mathrm{C} 6-\mathrm{C} 7-\mathrm{C} 8-\mathrm{C} 9$ & $179.9(3)$ & $\mathrm{S} 1-\mathrm{C} 31-\mathrm{C} 32-\mathrm{C} 33$ & $0.2(4)$ \\
\hline $\mathrm{C} 7-\mathrm{C} 8-\mathrm{C} 9-\mathrm{O} 2$ & $-3.1(5)$ & $\mathrm{C} 31-\mathrm{C} 32-\mathrm{C} 33-\mathrm{C} 34$ & $0.2(4)$ \\
\hline $\mathrm{C} 7-\mathrm{C} 8-\mathrm{C} 9-\mathrm{C} 10$ & $178.2(3)$ & $\mathrm{O} 5-\mathrm{C} 32-\mathrm{C} 33-\mathrm{C} 34$ & $178.6(3)$ \\
\hline $\mathrm{O} 2-\mathrm{C} 9-\mathrm{C} 10-\mathrm{C} 15$ & $140.7(3)$ & $\mathrm{C} 31-\mathrm{C} 32-\mathrm{C} 33-\mathrm{O} 6$ & $179.4(3)$ \\
\hline $\mathrm{C} 8-\mathrm{C} 9-\mathrm{C} 10-\mathrm{C} 15$ & $-40.5(5)$ & $\mathrm{O} 5-\mathrm{C} 32-\mathrm{C} 33-\mathrm{O} 6$ & $-2.2(5)$ \\
\hline $\mathrm{O} 2-\mathrm{C} 9-\mathrm{C} 10-\mathrm{C} 11$ & $-37.6(4)$ & $\mathrm{O} 6-\mathrm{C} 33-\mathrm{C} 34-\mathrm{S} 1$ & $-179.7(2)$ \\
\hline
\end{tabular}




\begin{tabular}{|c|c|}
\hline $\mathrm{C} 8-\mathrm{C} 9-\mathrm{C} 10-\mathrm{C} 11$ & $141.2(3)$ \\
\hline $\mathrm{C} 15-\mathrm{C} 10-\mathrm{C} 11-\mathrm{F} 6$ & $176.1(3)$ \\
\hline $\mathrm{C} 9-\mathrm{C} 10-\mathrm{C} 11-\mathrm{F} 6$ & $-5.5(5)$ \\
\hline $\mathrm{C} 15-\mathrm{C} 10-\mathrm{C} 11-\mathrm{C} 12$ & $-0.7(5)$ \\
\hline $\mathrm{C} 9-\mathrm{C} 10-\mathrm{C} 11-\mathrm{C} 12$ & $177.7(3)$ \\
\hline $\mathrm{F} 6-\mathrm{C} 11-\mathrm{C} 12-\mathrm{F} 7$ & $2.4(5)$ \\
\hline $\mathrm{C} 10-\mathrm{C} 11-\mathrm{C} 12-\mathrm{F} 7$ & $179.2(3)$ \\
\hline $\mathrm{F} 6-\mathrm{C} 11-\mathrm{C} 12-\mathrm{C} 13$ & $-177.5(3)$ \\
\hline $\mathrm{C} 10-\mathrm{C} 11-\mathrm{C} 12-\mathrm{C} 13$ & $-0.7(6)$ \\
\hline $\mathrm{F} 7-\mathrm{C} 12-\mathrm{C} 13-\mathrm{F} 8$ & $1.6(6)$ \\
\hline $\mathrm{C} 11-\mathrm{C} 12-\mathrm{C} 13-\mathrm{F} 8$ & $-178.4(3)$ \\
\hline $\mathrm{F} 7-\mathrm{C} 12-\mathrm{C} 13-\mathrm{C} 14$ & $-177.9(3)$ \\
\hline $\mathrm{C} 11-\mathrm{C} 12-\mathrm{C} 13-\mathrm{C} 14$ & $2.0(6)$ \\
\hline $\mathrm{F} 8-\mathrm{C} 13-\mathrm{C} 14-\mathrm{F} 9$ & $-0.7(6)$ \\
\hline $\mathrm{C} 12-\mathrm{C} 13-\mathrm{C} 14-\mathrm{F} 9$ & $178.9(4)$ \\
\hline $\mathrm{F} 8-\mathrm{C} 13-\mathrm{C} 14-\mathrm{C} 15$ & $178.5(3)$ \\
\hline $\mathrm{C} 12-\mathrm{C} 13-\mathrm{C} 14-\mathrm{C} 15$ & $-1.9(6)$ \\
\hline $\mathrm{F} 9-\mathrm{C} 14-\mathrm{C} 15-\mathrm{F} 10$ & $0.2(5)$ \\
\hline $\mathrm{C} 13-\mathrm{C} 14-\mathrm{C} 15-\mathrm{F} 10$ & $-179.0(3)$ \\
\hline $\mathrm{F} 9-\mathrm{C} 14-\mathrm{C} 15-\mathrm{C} 10$ & $179.7(3)$ \\
\hline $\mathrm{C} 13-\mathrm{C} 14-\mathrm{C} 15-\mathrm{C} 10$ & $0.5(6)$ \\
\hline $\mathrm{C} 11-\mathrm{C} 10-\mathrm{C} 15-\mathrm{F} 10$ & $-179.8(3)$ \\
\hline $\mathrm{C} 9-\mathrm{C} 10-\mathrm{C} 15-\mathrm{F} 10$ & $1.8(5)$ \\
\hline $\mathrm{C} 11-\mathrm{C} 10-\mathrm{C} 15-\mathrm{C} 14$ & $0.8(5)$ \\
\hline $\mathrm{C} 9-\mathrm{C} 10-\mathrm{C} 15-\mathrm{C} 14$ & $-177.6(3)$ \\
\hline $\mathrm{F} 11-\mathrm{C} 16-\mathrm{C} 17-\mathrm{F} 12$ & $-0.4(5)$ \\
\hline $\mathrm{C} 21-\mathrm{C} 16-\mathrm{C} 17-\mathrm{F} 12$ & $-179.4(3)$ \\
\hline $\mathrm{F} 11-\mathrm{C} 16-\mathrm{C} 17-\mathrm{C} 18$ & $178.4(3)$ \\
\hline $\mathrm{C} 21-\mathrm{C} 16-\mathrm{C} 17-\mathrm{C} 18$ & $-0.6(5)$ \\
\hline $\mathrm{F} 12-\mathrm{C} 17-\mathrm{C} 18-\mathrm{F} 13$ & $-1.0(5)$ \\
\hline $\mathrm{C} 16-\mathrm{C} 17-\mathrm{C} 18-\mathrm{F} 13$ & $-179.8(3)$ \\
\hline $\mathrm{F} 12-\mathrm{C} 17-\mathrm{C} 18-\mathrm{C} 19$ & $178.9(3)$ \\
\hline $\mathrm{C} 16-\mathrm{C} 17-\mathrm{C} 18-\mathrm{C} 19$ & $0.1(5)$ \\
\hline $\mathrm{F} 13-\mathrm{C} 18-\mathrm{C} 19-\mathrm{F} 14$ & $-0.4(5)$ \\
\hline $\mathrm{C} 17-\mathrm{C} 18-\mathrm{C} 19-\mathrm{F} 14$ & $179.7(3)$ \\
\hline $\mathrm{F} 13-\mathrm{C} 18-\mathrm{C} 19-\mathrm{C} 20$ & $-179.8(3)$ \\
\hline $\mathrm{C} 17-\mathrm{C} 18-\mathrm{C} 19-\mathrm{C} 20$ & $0.3(5)$ \\
\hline $\mathrm{F} 14-\mathrm{C} 19-\mathrm{C} 20-\mathrm{F} 15$ & $-1.9(5)$ \\
\hline $\mathrm{C} 18-\mathrm{C} 19-\mathrm{C} 20-\mathrm{F} 15$ & $177.5(3)$ \\
\hline $\mathrm{F} 14-\mathrm{C} 19-\mathrm{C} 20-\mathrm{C} 21$ & $-179.6(3)$ \\
\hline $\mathrm{C} 18-\mathrm{C} 19-\mathrm{C} 20-\mathrm{C} 21$ & $-0.1(5)$ \\
\hline $\mathrm{F} 15-\mathrm{C} 20-\mathrm{C} 21-\mathrm{C} 16$ & $-177.9(3)$ \\
\hline $\mathrm{C} 19-\mathrm{C} 20-\mathrm{C} 21-\mathrm{C} 16$ & $-0.4(5)$ \\
\hline $\mathrm{F} 15-\mathrm{C} 20-\mathrm{C} 21-\mathrm{C} 22$ & $-1.0(5)$ \\
\hline $\mathrm{C} 19-\mathrm{C} 20-\mathrm{C} 21-\mathrm{C} 22$ & $176.6(3)$ \\
\hline $\mathrm{F} 11-\mathrm{C} 16-\mathrm{C} 21-\mathrm{C} 20$ & $-178.2(3)$ \\
\hline $\mathrm{C} 17-\mathrm{C} 16-\mathrm{C} 21-\mathrm{C} 20$ & $0.8(5)$ \\
\hline $\mathrm{F} 11-\mathrm{C} 16-\mathrm{C} 21-\mathrm{C} 22$ & $4.8(5)$ \\
\hline
\end{tabular}

$\begin{array}{ll}\text { C32-C33-C34-S1 } & -0.5(4) \\ \text { O5-C35-C36-O6 } & 63.7(3) \\ \text { C31-C32-O5-C35 } & -165.6(3) \\ \text { C33-C32-O5-C35 } & 16.2(4) \\ \text { C36-C35-O5-C32 } & -45.5(4) \\ \text { C34-C33-O6-C36 } & -162.4(3) \\ \text { C32-C33-O6-C36 } & 18.6(4) \\ \text { C34-C33-O6-Cu1 } & 42.2(4) \\ \text { C32-C33-O6-Cu1 } & -136.9(3) \\ \text { C35-C36-O6-C33 } & -47.5(3) \\ \text { C35-C36-O6-Cu1 } & 107.8(3) \\ \text { C32-C31-S1-C34 } & -0.4(3) \\ \text { C33-C34-S1-C31 } & 0.5(3) \\ \text { S2A-C37A-C38A-O7A } & 177.3(4) \\ \text { S2A-C37A-C38A-C39A } & -2.5(4) \\ \text { O7A-C38A-C39A-C40A } & -179.5(3) \\ \text { C37A-C38A-C39A-C40A } & 0.3(2) \\ \text { O7A-C38A-C39A-O8A } & 0.5(3) \\ \text { C37A-C38A-C39A-O8A } & -179.7(2) \\ \text { O8A-C39A-C40A-S2A } & -178.3(4) \\ \text { C38A-C39A-C40A-S2A } & 1.8(4) \\ \text { O7A-C41A-C42A-O8A } & 62.1(9) \\ \text { C39A-C38A-O7A-C41A } & 16.2(6) \\ \text { C37A-C38A-O7A-C41A } & -163.6(5) \\ \text { C42A-C41A-O7A-C38A } & -45.8(8) \\ \text { C40A-C39A-O8A-C42A } & -165.9(6) \\ \text { C38A-C39A-O8A-C42A } & 14.1(6) \\ \text { C41A-C42A-O8A-C39A } & -44.6(8) \\ \text { C38A-C37A-S2A-C40A } & 3.0(4) \\ \text { C39A-C40A-S2A-C37A } & -2.8(5) \\ \text { S2B-C37B-C38B-O7B } & 177.9(5) \\ \text { S2B-C37B-C38B-C39B } & -2.2(5) \\ \text { C37B-C38B-C39B-O8B } & 179.6(3) \\ \text { O7B-C38B-C39B-O8B } & -0.5(5) \\ \text { C37B-C38B-C39B-C40B } & -0.1(3) \\ \text { O7B-C38B-C39B-C40B } & 179.8(4) \\ \text { O8B-C39B-C40B-S2B } & -177.2(6) \\ \text { C38B-C39B-C40B-S2B } & 2.5(6) \\ \text { O7B-C41B-C42B-O8B } & 67.4(17) \\ \text { C37B-C38B-O7B-C41B } & -171.9(10) \\ \text { C39B-C38B-O7B-C41B } & 8.2(11) \\ \text { C42B-C41B-O7B-C38B } & -42.0(16) \\ \text { C40B-C39B-O8B-C42B } & -156.4(10) \\ \text { C38B-C39B-O8B-C42B } & 23.9(10) \\ \text { C41B-C42B-O8B-C39B } & -53.7(14) \\ \text { C39B-C40B-S2B-C37B } & -3.1(7) \\ \text { C38B-C37B-S2B-C40B } & 3.1(7) \\ \text { S3-C43-C44-O9 } & 179.3(3) \\ & \end{array}$




$\begin{array}{llll}\mathrm{C} 17-\mathrm{C} 16-\mathrm{C} 21-\mathrm{C} 22 & -176.2(3) & \mathrm{S} 3-\mathrm{C} 43-\mathrm{C} 44-\mathrm{C} 45 & -2.8(5) \\ \mathrm{C} 20-\mathrm{C} 21-\mathrm{C} 22-\mathrm{O} 3 & -124.5(3) & \mathrm{O} 9-\mathrm{C} 44-\mathrm{C} 45-\mathrm{C} 46 & 179.6(4) \\ \mathrm{C} 16-\mathrm{C} 21-\mathrm{C} 22-\mathrm{O} 3 & 52.3(4) & \mathrm{C} 43-\mathrm{C} 44-\mathrm{C} 45-\mathrm{C} 46 & 1.7(6) \\ \mathrm{C} 20-\mathrm{C} 21-\mathrm{C} 22-\mathrm{C} 23 & 54.3(4) & \mathrm{O} 9-\mathrm{C} 44-\mathrm{C} 45-\mathrm{O} 10 & -0.1(6) \\ \mathrm{C} 16-\mathrm{C} 21-\mathrm{C} 22-\mathrm{C} 23 & -129.0(3) & \mathrm{C} 43-\mathrm{C} 44-\mathrm{C} 45-\mathrm{O} 10 & -178.1(4) \\ \mathrm{O} 3-\mathrm{C} 22-\mathrm{C} 23-\mathrm{C} 24 & -3.4(6) & \mathrm{O} 10-\mathrm{C} 45-\mathrm{C} 46-\mathrm{S} 3 & 0.4(6) \\ \mathrm{C} 21-\mathrm{C} 22-\mathrm{C} 23-\mathrm{C} 24 & 178.0(3) & \mathrm{C} 44-\mathrm{C} 45-\mathrm{C} 46-\mathrm{S} 3 & 61.2(4) \\ \mathrm{C} 22-\mathrm{C} 23-\mathrm{C} 24-\mathrm{O} 4 & -6.9(5) & \mathrm{O} 9-\mathrm{C} 47-\mathrm{C} 48-\mathrm{O} 10 & -169.8(4) \\ \mathrm{C} 22-\mathrm{C} 23-\mathrm{C} 24-\mathrm{C} 25 & 171.6(3) & \mathrm{C} 43-\mathrm{C} 44-\mathrm{O} 9-\mathrm{C} 47 & 12.5(5) \\ \mathrm{O} 4-\mathrm{C} 24-\mathrm{C} 25-\mathrm{C} 26 & -42.2(4) & \mathrm{C} 45-\mathrm{C} 44-\mathrm{O} 9-\mathrm{C} 47 & -162.9(4) \\ \mathrm{C} 23-\mathrm{C} 24-\mathrm{C} 25-\mathrm{C} 26 & 139.1(3) & \mathrm{C} 46-\mathrm{C} 45-\mathrm{O} 10-\mathrm{C} 48 & 16.9(5) \\ \mathrm{O} 4-\mathrm{C} 24-\mathrm{C} 25-\mathrm{C} 30 & 132.2(3) & \mathrm{C} 44-\mathrm{C} 45-\mathrm{O} 10-\mathrm{C} 48 & -44.6(5) \\ \mathrm{C} 23-\mathrm{C} 24-\mathrm{C} 25-\mathrm{C} 30 & -46.4(4) & \mathrm{C} 47-\mathrm{C} 48-\mathrm{O} 10-\mathrm{C} 45 & -1.7(4) \\ \mathrm{C} 30-\mathrm{C} 25-\mathrm{C} 26-\mathrm{F} 16 & 178.4(3) & \mathrm{C} 45-\mathrm{C} 46-\mathrm{S} 3-\mathrm{C} 43 & 2.6(4) \\ \mathrm{C} 24-\mathrm{C} 25-\mathrm{C} 26-\mathrm{F} 16 & -6.8(5) & \mathrm{C} 44-\mathrm{C} 43-\mathrm{S} 3-\mathrm{C} 46 & \\ \mathrm{C} 30-\mathrm{C} 25-\mathrm{C} 26-\mathrm{C} 27 & 0.4(5) & & \end{array}$

Symmetry code: (i) $x+1, y, z$.

Hydrogen-bond geometry $\left(\AA,{ }^{o}\right)$

\begin{tabular}{lllll}
\hline$D-\mathrm{H} \cdots A$ & $D-\mathrm{H}$ & $\mathrm{H} \cdots A$ & $D \cdots A$ & $D-\mathrm{H} \cdots A$ \\
\hline $\mathrm{C} 23-\mathrm{H} 23 \cdots \mathrm{F} 17^{\mathrm{ii}}$ & 0.95 & 2.41 & $3.362(4)$ & 179 \\
$\mathrm{C} 31-\mathrm{H} 31 \cdots \mathrm{O} 1^{\mathrm{iii}}$ & 0.95 & 2.57 & $3.351(4)$ & 139 \\
$\mathrm{C} 35-\mathrm{H} 35 A \cdots \mathrm{O} 8 B^{\text {iii }}$ & 0.99 & 2.45 & $3.349(16)$ & 151 \\
$\mathrm{C} 37 A-\mathrm{H} 37 A \cdots \mathrm{S} 1^{\text {iv }}$ & 0.95 & 2.77 & $3.590(9)$ & 145 \\
$\mathrm{C} 41 A-\mathrm{H} 41 A \cdots \mathrm{S} 2 A^{\mathrm{i}}$ & 0.99 & 2.51 & $3.051(11)$ & 114 \\
$\mathrm{C} 42 A-\mathrm{H} 42 A \cdots \mathrm{S} 2 A^{\mathrm{i}}$ & 0.99 & 2.57 & $3.220(9)$ & 123 \\
$\mathrm{C} 42 A-\mathrm{H} 42 A \cdots \mathrm{F} 6^{\mathrm{i}}$ & 0.99 & 2.45 & $3.162(8)$ & 128 \\
$\mathrm{C} 48-\mathrm{H} 48 B \cdots \mathrm{F} 10^{\mathrm{v}}$ & 0.99 & 2.51 & $3.326(5)$ & 140 \\
\hline
\end{tabular}

Symmetry codes: (i) $x+1, y, z$; (ii) $x,-y+3 / 2, z-1 / 2$; (iii) $x-1, y, z$; (iv) $x, y, z-1$; (v) $-x+1,-y+1,-z+1$. 